\title{
Refractometric Properties of a TFBG Sensor Demodulated Using $\alpha$-Shape Modified Delaunay Triangulation
}

\author{
Luigi Fazzi *(D) and Roger M. Groves \\ Structural Integrity and Composites Group, Faculty of Aerospace Engineering, Delft University of Technology, \\ 2629 HS Delft, The Netherlands; r.m.groves@tudelft.nl \\ * Correspondence: 1.fazzi@tudelft.nl
}

\begin{abstract}
In our previous research, a novel demodulation technique based on $\alpha$-shape Delaunay triangulation (D-T) was developed to obtain the refractive index of the medium surrounding the optical fibre using the envelope of the cladding peaks from the spectrum of the tilted fibre Bragg grating (TFBG) sensor. This technique was demonstrated to be efficient, easy to implement, powerful, faster than the previous ones and applicable for real-time measurements. In this paper, a deep parametric analysis of the resolution, repeatability and accuracy of the D-T demodulation technique for a TFBG refractometer sensor is performed and presented. The spectral properties of the TFBG sensor as a refractometer are explored using the same demodulation technique. Specifically, supposing the use of the TFBG as a two-parameter optical sensor, the influence of the strain on the envelope area is analysed, and the measurement stability regarding the external RI is reported. Then, the cladding resonance peaks in the spectrum are observed experimentally as the TFBG undergoes partial immersion in a defined refractive index liquid. This last experiment allowed a better understanding the evolution of the TFBG transmission spectrum when the Bragg gratings were partially surrounded by a medium with a different RI.
\end{abstract}

Citation: Fazzi, L.; Groves, R.M.

Refractometric Properties of a TFBG Sensor Demodulated Using $\alpha$-Shape Modified Delaunay Triangulation. Optics 2021, 2, 113-133. https:// doi.org/10.3390/opt2020012

Academic Editor:

Francesco Chiavaioli

Received: 19 May 2021

Accepted: 2 June 2021

Published: 9 June 2021

Publisher's Note: MDPI stays neutral with regard to jurisdictional claims in published maps and institutional affiliations.

Copyright: (c) 2021 by the authors. Licensee MDPI, Basel, Switzerland. This article is an open access article distributed under the terms and conditions of the Creative Commons Attribution (CC BY) license (https:/ / creativecommons.org/licenses/by/ $4.0 /)$.
Keywords: Delaunay triangulation; tilted fibre Bragg grating; FBG; demodulation; refractometer; refractive index; sensor

\section{Introduction}

The customization of the Bragg grating structure allows special spectral features to be obtained that can be exploited for sensing and measurement applications. Fibre Bragg gratings (FBGs) are manufactured through a permanent modulation of the core RI with a given profile (uniform, Gaussian, chirped, apodaizing and superstructure) [1]. Currently, the most practised FBG manufacturing technique is based on the use of a phase mask to apply the RI profile variation in the core of the optical fibre (OF). A tilted FBG can be manufactured by tilting the $\mathrm{OF}$, the phase mask or the laser beam or by a combination of these. In this way, the RI modulation is super-imposed with an inclination with respect to the optical axis in the OF core.

This OF sensor is called a tilted FBG due to the imposition of a tilt angle to the Bragg grating structure. The special features of the tilted fibre Bragg grating (TFBG) spectrum offer multi-sensing capabilities [2] and make this sensor versatile while maintaining the same benefits as the standard FBG sensor. The use of the TFBG as a refractometer is one possible application. In fact, the modes-coupling system is sensitive to the surrounding RI [3]; hence, some resonance peaks in the transmission spectrum can be used to measure the RI variations. With this aim, several techniques have been developed, which can be divided into the methods that use wavelength-encoded information and those techniques that consider the TFBG transmission power (global methods).

Exploiting these demodulation techniques, the TFBG sensing ability toward the surrounding RI variation has been investigated in several applications, such as the monitoring of the cure degree of the resin during composite manufacturing [4,5], the microfluidic 
rate [6] and in chemical and biochemical fields [7-10]. However, since these previous demodulation techniques suffer certain drawbacks and limitations, in a previous work [11], we developed a new demodulation approach based on Delaunay triangulation (D-T) to obtain the surrounding RI value from the envelope area of the cladding modes.

The technique was demonstrated to be simple and fast to implement, saving time and reducing the computational power required, flexible, insensitive to variations in the spectrum shape and compatible for real-time measurements and simultaneous multiple measurements. However, this previous work was focused on the algorithm of the demodulation technique. Here, a parametric analysis is achieved on the RI measurement performance (the stability, resolution and accuracy) of the TFBG sensor by performing the D-T demodulation of the TFBG spectra under full immersion for a wide range of RIs. In this way, the performance of the technique is fully analysed based on the fundamental $\alpha$-parameter used to perform the demodulation.

An equally important aspect is the strain cross-sensitivity effect on the RI measurements performing the D-T technique. Indeed, although a temperature variation implies a blue- or red-shift of the entire TFBG transmission spectrum and an amplitude change of the cladding peaks, it has been demonstrated this does not have a direct effect on the RI measurements using the global demodulation techniques [3,12]. However, the strain cross-sensitivity when using the novel D-T method has not yet been tested for detecting the variation of the cladding peak envelope area when the TFBG is immersed into three different RI liquids.

Indeed, as the D-T technique is compatible to be used simultaneously with other techniques for multi-parameter measurements, this investigation is important to assess a cross-sensitivity influence in measuring different parameters. In previous literature on the TFBG as refractometer sensor, the technique was assessed when fully immersed in a RI liquid. However, advanced applications [4,5] may involve a temporary or permanent partial immersion of the TFBG in a medium with a different RI. In this case, the sensor would be surrounded from different RIs, which would generate an unknown evolution of the cladding peaks envelope area.

Therefore, to make up this gap, this problem was investigated by partially dipping the TFBG into a RI oil and analysing the evolution of the spectra recorded during the immersion. This allowed us to obtain important information on the refractometric behaviour of the cladding resonance peaks, which can be exploited in applications where the TFBG is partially immersed. All these topics are fundamental for a complete characterisation and understanding of the D-T demodulation technique applied to the TFBG sensor for refractometric purposes.

First of all, the TFBG refractometric theory is presented in Section 2, while the experimental setup used during the entire testing campaign is described in Section 3. Then, the investigation of the strain cross-sensitivity on the RI measurement is reported in Section 4 and, following this, the RI measurement performance is treated in Sections 4-6. Finally, our analysis regarding the partial TFBG immersion is presented in Section 7.

\section{TFBG Theory and Influence of the Surrounding RI}

Tilted short-period Bragg gratings are a special kind of FBG where the permanent modulation of the core RI is super-imposed with an inclination with respect to the optical axis of the fibre. This so-called tilt angle allows the enhancement of the cladding modes coupling with the fundamental forward-propagating core mode [13]. Therefore, unlike standard FBGs, in the TFBG transmission spectrum, there are many close resonance peaks distributed along wavelengths smaller than the wavelength of the Bragg peak. Actually, other FBG types also have these in their transmission spectrum; however, due to cladding resonances losses, they are very weak compared to the propagating modes.

As it can be understood, the tilt angle influences the mode-coupling inside the OF as well as the shape of the spectrum. In the case of weakly tilted FBGs, where the maximum angle is $\sim 15^{\circ}$, the transmission signal is characterized by the Bragg, the Ghost and the 
cladding resonance peaks (Figure 1). Though not used in this work, TFBGs with higher tilt angles can also be used for refractometric purposes [3]; however, the Ghost resonance is missing in their spectrum and the Bragg peak is attenuated. Since, the cladding resonances change their amplitude and wavelength with the surrounding RI variation, it is important that the detection of the Ghost and Bragg peaks uses the TFBG as a three-parameter sensor.

The Bragg peak is obtained through the core-core mode coupling, while the cladding peaks are generated by the coupling between the core forward-propagating mode and the cladding backward-propagating modes. The Ghost peak can be explained as the coupling between cladding modes with a low interaction with the interface between the cladding and the surrounding but propagating in a well-confined region of the cladding layer with a strong interaction with the fibre core. From their nature, the Bragg and the Ghost peaks are sensitive to thermo-mechanical perturbations but not to external RI variations, unlike the cladding resonances, which are susceptible to the surrounding RI in amplitude and in wavelength position $[3,14]$.

In this work, only weakly TFBG were used because the special behaviour of the different peaks makes the weakly tilted FBG most suitable as a sensor for three-parameter sensing applications [11]. Figure 2 shows the internal structure of a weakly tilted FBG, where the tilt angle $\theta$, the grating period $\Lambda_{\mathrm{G}}$ along the OF axis and the nominal grating period $\Lambda=\Lambda_{\mathrm{G}} \cos \theta$ are defined. By considering the effective refractive index $n_{\text {eff }, i}$ of each mode, the nominal wavelength can be calculated for each peak in the spectrum. Specifically $\mathrm{n}_{\text {eff,core }}$ and $\mathrm{n}_{\mathrm{eff}, \mathrm{clad}, i}$ are respectively referred to as the core and cladding modes. The wavelengths of the Bragg $\left(\lambda_{\text {Bragg }}\right)$ and cladding $\left(\lambda_{\text {clad }, i}\right)$ resonance peaks can be then obtained using the following fundamental equations [15]:

$$
\begin{gathered}
\lambda_{\text {Bragg }}=2 \mathrm{n}_{\text {eff,core }} \Lambda=2 \mathrm{n}_{\text {eff,core }} \frac{\Lambda_{\mathrm{G}}}{\cos \theta}, \\
\lambda_{\text {clad }, i}=\left(\mathrm{n}_{\text {eff,core }}+\mathrm{n}_{\text {eff,clad }, i}\right) \Lambda=2\left(\mathrm{n}_{\text {eff,core }}+\mathrm{n}_{\mathrm{eff}, \mathrm{clad}, i}\right) \frac{\Lambda_{\mathrm{G}}}{\cos \theta} .
\end{gathered}
$$

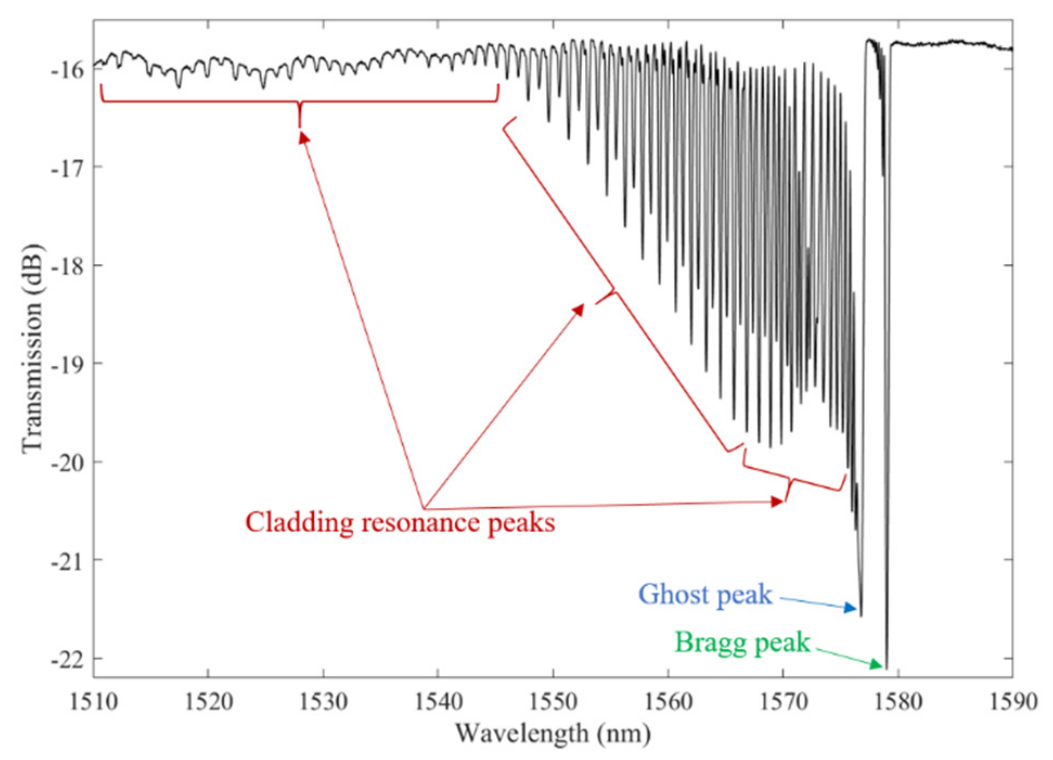

Figure 1. The $3^{\circ}$ tilted Fibre Bragg Grating (TFBG) transmission spectrum. 
Cladding

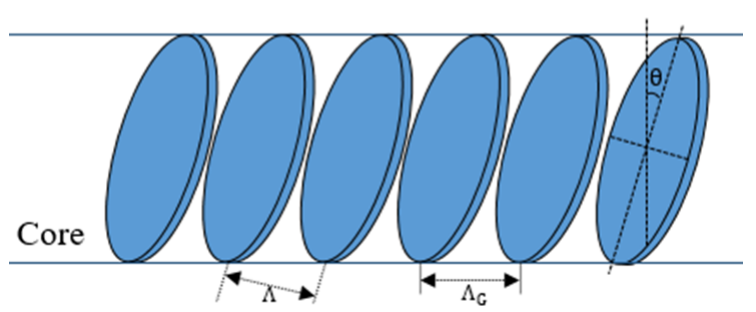

Figure 2. Schematic of a weakly TFBG internal structure.

Once the Bragg structure is defined, if no mechanical perturbations are applied to the $\mathrm{OF}$, then the grating period and the tilt angle are constant, then $\mathrm{n}_{\mathrm{eff}, \mathrm{core}}$ is also fixed because the RI of the core and cladding OF layers are defined. As $\mathrm{n}_{\mathrm{eff}, \mathrm{clad}, i}$ is sensitive to the surrounding RI variations, each cladding mode has to fit with a new propagating condition so that the phase-matching condition (represented by Equation (2)) is satisfied and the nominal wavelength changes for each external RI.

In the same way, even the mode amplitude is influenced when the surrounding RI becomes closer to the effective RI of the $i$-th mode because it becomes less guided within the cladding and its resonances become weaker. At a certain point, when the external RI matches the effective RI of $i$-th mode, the mode is irradiated out from the OF. This happens because the mode and the surrounding medium have the same refractive index, as there is no optical boundary between the two layers, and the internal reflection is missing.

As a consequence, the relative resonance does not appear any longer in the spectrum. Based on the RI effects on the amplitude and wavelength of the TFBG cladding peaks, several techniques have been developed to correlate the TFBG signal with the surrounding RI measurements. To obtain the best measurement performance using a novel approach based on the Delaunay triangulation, an investigation into the statistical and sensing aspects has to be performed, to assess the effect of a strain perturbation on the envelope area and a parametric analysis needs to be performed to determine the measurement stability of the technique.

\section{Experimental Setup for RI Measurement Performance and TFBG Immersion Test}

In this section the experimental setup and the TFBG sensor used to perform the RI measurement performance and the immersion test are presented. In particular, a $3^{\circ}$ tilted FBG, written in a PS1250/1500 FiberCore standard OF by FORC Photonics company, was placed centred in a dedicated linear translation stage (Figure 3), where, in the middle, a set of oil liquids, produced by Cargille labs (with the RI accuracy estimated as \pm 0.0002 [16]), was used for the fibre optic sensor immersion.

The RI liquid was placed on top of a polycarbonate surface. A K-thermocouple (TC), with accuracy of $\pm 1^{\circ} \mathrm{C}$, was placed close to the immersion point to check the temperature. The accuracy of the translation stage $( \pm 5 \mu \mathrm{m}$ [17]) depends on its linear electric actuator. The linear translation stage has the double task of holding the OF straight and in position and inducing axial deformations.

The ends of the OF were connected to an optical circulator, which, in turn, was linked to a single input/output channel of a NI-PXIe 4844 FBG interrogator, whose wavelength range was 1510-1590 $\mathrm{nm}$ with scanning resolution of $4 \mathrm{pm}$ ( $\pm 1 \mathrm{pm}$ accuracy) and minimum power detection of $6.103 \times 10^{-4} \mathrm{dBm}$. The vertical translation stage was used to perform the full and partial immersion tests as described in Section 8. 


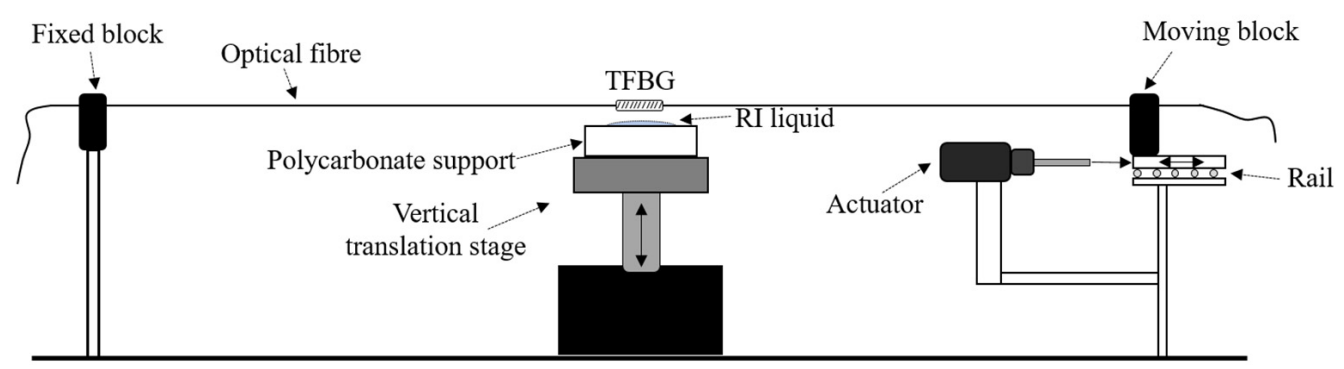

Figure 3. Schematic of the experimental setup dedicated to the strain effect investigation.

\section{RI Calibration Curve and Strain Cross-Sensitivity on RI Measurements}

First of all, the RI correlation curve was obtained using the setup in Figure 3 by applying a small pretension displacement of $\sim 30 \mu \mathrm{m}$ on the OF. The TFBG spectra were demodulated using an $\alpha$-shape D-T parameter $(\alpha)$ of 2 , and the reference envelope area was considered at 1.33 RI. The resulting correlation curve is reported in Figure 4.

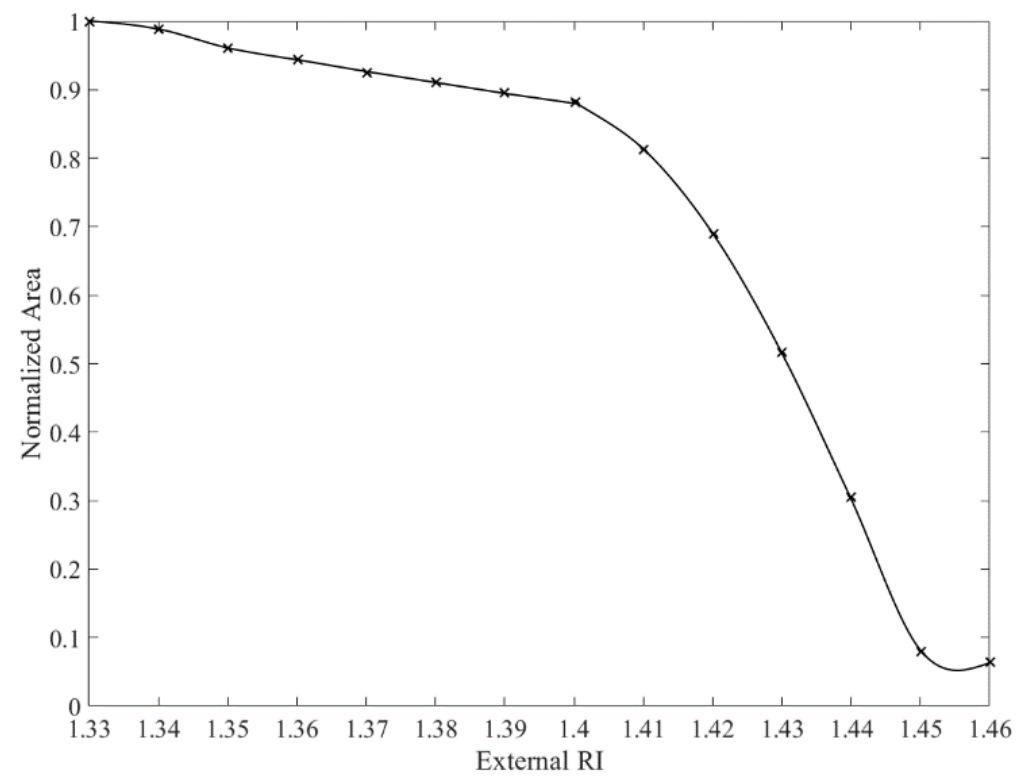

Figure 4. The correlation spline-curve between surrounding refractive indices (RIs) and cladding peaks envelope areas of the $3^{\circ}$ tilted FBG.

Once the calibration has been completed, the cross-sensitivity of the RI measurements was tested using the same setup and pretension deformation. Therefore, the TFBG was fully immersed in 1.33, 1.40 and $1.46 \mathrm{RI}$ liquids and underwent several levels of axial deformation. The spectra were recorded every $50 \mu \varepsilon$ up to a maximum value of $1000 \mu \varepsilon$. The cladding resonance envelope areas, obtained with the D-T technique, were normalized with the same reference area of the RI calibration.

The experiment was performed at a room temperature of $22^{\circ} \mathrm{C}$; however, the TC in the immersion point was detecting temperature oscillations of $\pm 2{ }^{\circ} \mathrm{C}$ during the test. Once the TFBG was completely immersed in each RI liquid, the translation stage was moved to induce the deformation to the OF, and the TFBG spectrum was recorded. The displacements were given as inputs to the linear actuator through computer software (provided by the manufacturer), while the spectra were acquired with a dedicated LabView code. The envelope area trends are shown in Figure 5. 


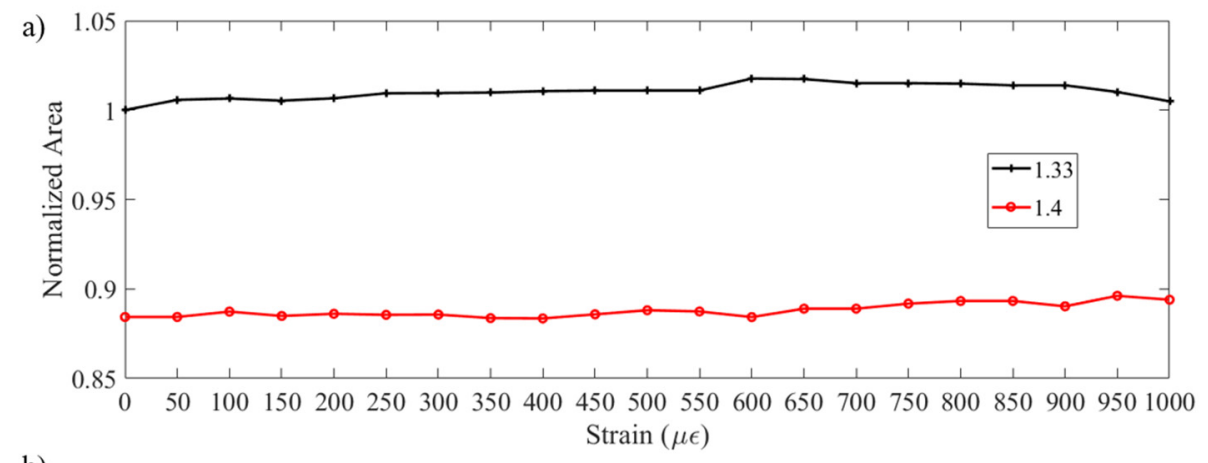

b)

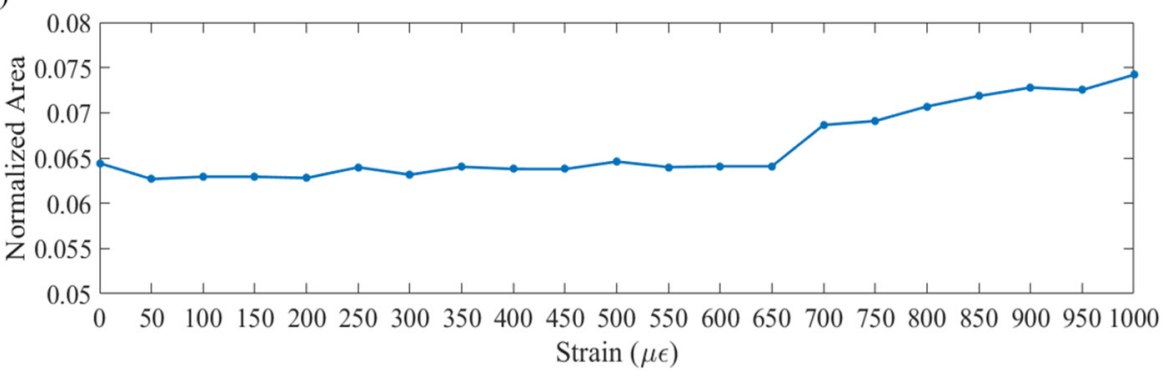

Figure 5. (a) Trends of the normalized areas for 1.33 and 1.40 RIs and (b) 1.46, with the increasing of strain on the TFBG sensor.

From Figure 5, the variation of the normalized area with the increase of the strain is negligible, as the trends are almost flat along the applied strain values. The maximum detected area variation along all of the strain range is $1.76 \%$ when considering $\alpha=2$ for the 1.33 curve. Now, considering the worst sensitivity range of the TFBG (observable in Figure 4), which is between 1.33-1.40, and the linear fitting correlation function, the maximum RI error measurement caused by the strain perturbation is 0.0103 . This value is calculated by considering the worst measurement condition without removing the influence of the temperature changes on the RI and the fitting error.

At this point, the temperature variations during the test and the $\frac{d R I}{d T}$ coefficient (RI variation with respect to the temperature variation) of the immersion liquids are taken into account. Hence, considering the temperature at the maximum RI error (obtained at $600 \mu \varepsilon)$ when the TFBG is immersed in the $1.33 \mathrm{RI}$ liquid $\left(\frac{\mathrm{dRI}}{\mathrm{dT}}=4.5 \times 10^{-4}{ }^{\circ} \mathrm{C}^{-1}\right.$ in the $15-35{ }^{\circ} \mathrm{C}$ range), the RI variation caused by the temperature variation can be removed from the total error to obtain a new value of $8.05 \times 10^{-3}$. For the same RI liquid, this RI variation remains almost stable until $900 \mu \varepsilon$, while when considering the total strain range, an average error value of $2.4 \times 10^{-3}$ is obtained.

Following the same procedure, when the TFBG sensor is immersed in the oils with a 1.40 and 1.46 nominal RI, the calculated average RI error values are $9.6 \times 10^{-4}$ and $1.05 \times 10^{-3}$, respectively. However, as Figure 5 shows, for each liquid used, the trends are substantially flat starting from 0 until $600 \mu \varepsilon$, where the RI variations due to the strain perturbation are comparable with the coefficient $\frac{\mathrm{dRI}}{\mathrm{dT}}$ of the immersion liquids $\left(3.0 \times 10^{-4}\right.$ $\left.4.5 \times 10^{-4}\right)$.

If a general application of these sensors is considered, and looking at the operating temperature range of Bragg gratings written in silica OF without coating protection, which is broad $\left(-100{ }^{\circ} \mathrm{C}-900{ }^{\circ} \mathrm{C}\right)[18,19]$, the temperature influence on the RI variation is expected to be far greater than the strain perturbation in a real measurement application. Moreover, all errors reported here include the possible deviations induced in the spectrum from the precision in amplitude and wavelength scanning of the interrogation system.

\section{Stability of the $\alpha$-Shape Modified Delaunay Demodulation Technique}

In this section, a study of the robustness of the $\alpha$-shape modified Delaunay triangulation in measuring the external RI from the TFGB spectra is reported. For this purpose, the 
same experimental setup of Figure 3 was used, where the OF was fixed to the linear translation stage with a pre-tension of $\sim 100 \mu \mathrm{m}$. The TFBG spectrum was recorded 10 times for each RI liquid, and more specifically the OF sensor was cleaned with acetone and allowed to dry after each immersion. In this way, each TFBG measurement was independent of the previous one, even in the case where the RI liquid was the same between two consecutive measurements.

Therefore, each envelope area is related to the correspondent RI liquid where the sensor was immersed. In Figure 6, the blue circles are the correlation points between the calculated envelope areas and the surrounding RI liquid. The analysis was conducted for a RI liquid range from 1.40 until 1.60. This range includes the maximum refractometry sensitivity range (1.40-1.46) of the TFBG, and furthermore demonstrates that the sensor was able to function even at higher RIs $(>1.46)$, where all the cladding modes should have been irradiated out of the OF.

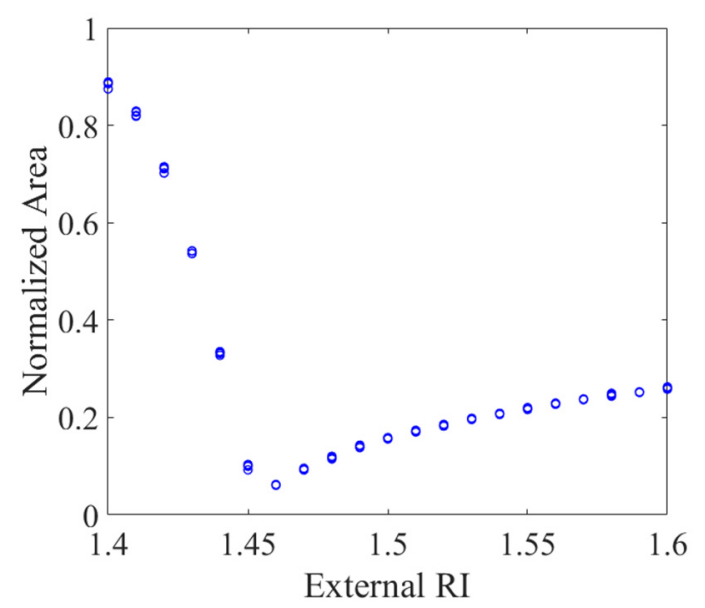

Figure 6. Ten correlation points measured with TFBG sensor for each used RI liquid.

This is possible, as part of the light continues to be irradiated externally, while some cladding modes return to be reflected internally towards the OF core where they are coupled [11]. Previous work that studied the TFBG sensor as a refractometer [3,12,20-22], did not take this RI range into account during their analysis. In some monitoring applications [4], the TFBG sensor is defined as not able to detect the resin's RI due to the lower RI of the OF. However, as demonstrated in Figure 6, these claims appear not to respect the actual behaviour of the OF sensor.

Indeed, though with a reduced sensitivity, the envelope area continues to be sensitive to the external RI. This means that the TFBG sensors can potentially be used to detect the surrounding RIs also in this range. At this point, the mean value $\mu$ was calculated for each set of $10 \mathrm{RI}$ correlation points. In the meantime, to estimate the spread of the measurements, the $\sigma$ standard deviation was also calculated in order to determine the reliability and repeatability of the D-T demodulation technique. The mean values are reported in Figure 7a,b as black lines with the corresponding red dashed-lines as limits that represent the $\sigma$ distance with respect to the average values.

Specifically, these curves are determined by performing a cubic spline interpolation by considering the $\sigma$ upper and lower limits and the average values. In Figure 7a, the $\sigma$ value is greater for lower RI liquids, while, for higher RI liquids, the black lines are closer to the mean values. In particular, the $\sigma$ curves approach closer to the black curve of the interpolated average values with increasing RI, up to almost coincide in some parts of the graph.

Figure $7 \mathrm{~b}$ shows the same inverted behaviour, where, for lower RIs, the $\sigma$ curves are closer to the black line and then widen for higher RI values. That could indicate that the TFBG spectrum is more stable when most of the cladding resonances are not present in the transmission signal. This analysis also demonstrates that the D-T demodulation 
technique offers excellent characteristics of reliability and repeatability in transducing the TFBG spectrum into a RI measurement. For clarity, the mean and the standard deviation values used to make the graphs in Figure 7a,b are reported in Table 1 for each RI.
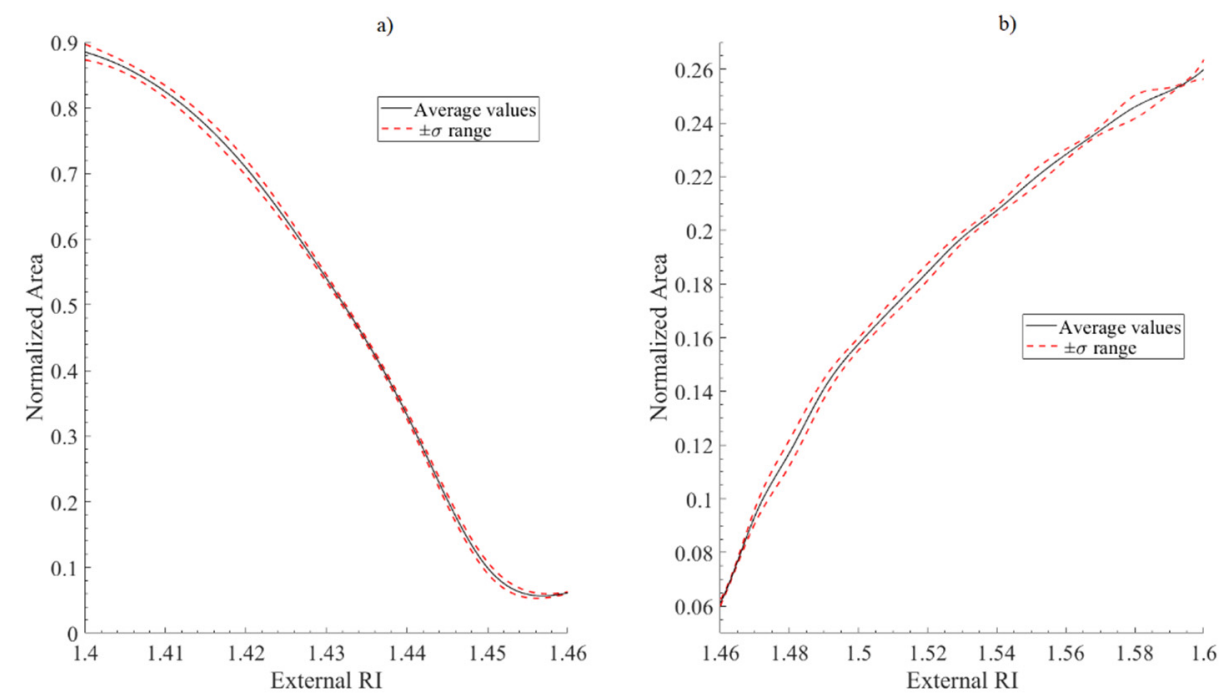

Figure 7. Cubic spline interpolation curves of the average and upper and lower standard deviation values in the RI range (a) 1.40-1.46 and (b) 1.46-1.60.

Table 1. Values of the mean and standard deviation for the 10 set points at each RI.

\begin{tabular}{ccc}
\hline RI & $\boldsymbol{\mu}$ & $\boldsymbol{\sigma}$ \\
\hline 1.40 & 0.8850 & 0.0060 \\
1.41 & 0.8244 & 0.0046 \\
1.42 & 0.7085 & 0.0061 \\
1.43 & 0.5399 & 0.0028 \\
1.44 & 0.3322 & 0.0030 \\
1.45 & 0.0997 & 0.0043 \\
1.46 & 0.0614 & 0.0006 \\
1.47 & 0.0937 & 0.0014 \\
1.48 & 0.1172 & 0.0024 \\
1.49 & 0.1410 & 0.0018 \\
1.50 & 0.1577 & 0.0012 \\
1.51 & 0.1713 & 0.0014 \\
1.52 & 0.1845 & 0.0016 \\
1.53 & 0.1973 & 0.0010 \\
1.54 & 0.2074 & 0.0008 \\
1.55 & 0.2183 & 0.0016 \\
1.56 & 0.2282 & 0.0010 \\
1.57 & 0.2372 & 0.0007 \\
1.58 & 0.2459 & 0.0022 \\
1.59 & 0.2518 & 0.0006 \\
1.60 & 0.2599 & 0.0018 \\
\hline
\end{tabular}

As Figure 7a,b and Table 1 show, it is possible to note that, for RIs greater than 1.46 , the standard deviations always fluctuated around the same values, while the maximum values were obtained from 1.4 to 1.42. A possible reason for this greater deviation at lower RIs could derive from the presence in the spectrum of cladding resonances whose peaks are not well defined but appear as double or triple peaks. In fact, as previously introduced, though these weakly guided cladding modes tend to disappear with increasing surrounding RI, they are still visible in the TFBG transmitted spectrum for RI liquids between 1.40-1.42.

The degeneration of the single peak into multiple peaks is due to the splitting of the vector modes as their electrical propagation field is less confined in the cladding layer, to 
the point that the linearly polarized mode approximations are no longer valid to describe the coupling mode theory [15]. The sensing characteristic of these higher cladding modes to the strain and RI variations is such that they have a large irregular wavelength shift and sensitivity coefficients [23].

This means that small external perturbations generate large non-uniform variations of the relative peaks in the spectrum, which are difficult to monitor due to the splitting of the peak. This effect was noted when comparing the TFBG transmission spectra of two subsequent measurements using the same RI liquid. In Figure 8, these two consecutive TFBG spectra using 1.40 RI liquid are shown, zoomed-in on the higher cladding resonance peaks.

These peaks are easily identified (wavelength range of 1541-1547 nm), not only by the irregular shape of the peak but also by the abrupt decay of their amplitude in the spectrum compared to the other peaks. From Figure 8, it is possible to note that the shapes of the upper and lower peaks between two consecutive spectra, recorded at the same experiment conditions, were different and not uniform. At this point, performing the D-T demodulation on both the spectra and focusing on the same wavelength range, the area regions created by the triangulation were dissimilar between the curves as it is possible to note from Figure 9.

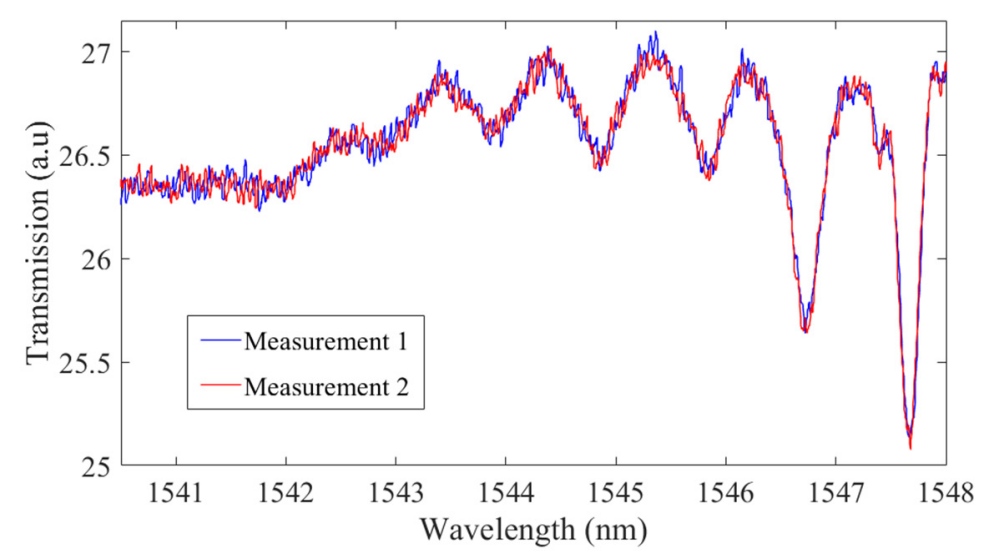

Figure 8. Higher cladding resonance peaks in the TFBG spectrum of two consecutive measurements using 1.40 RI liquid.

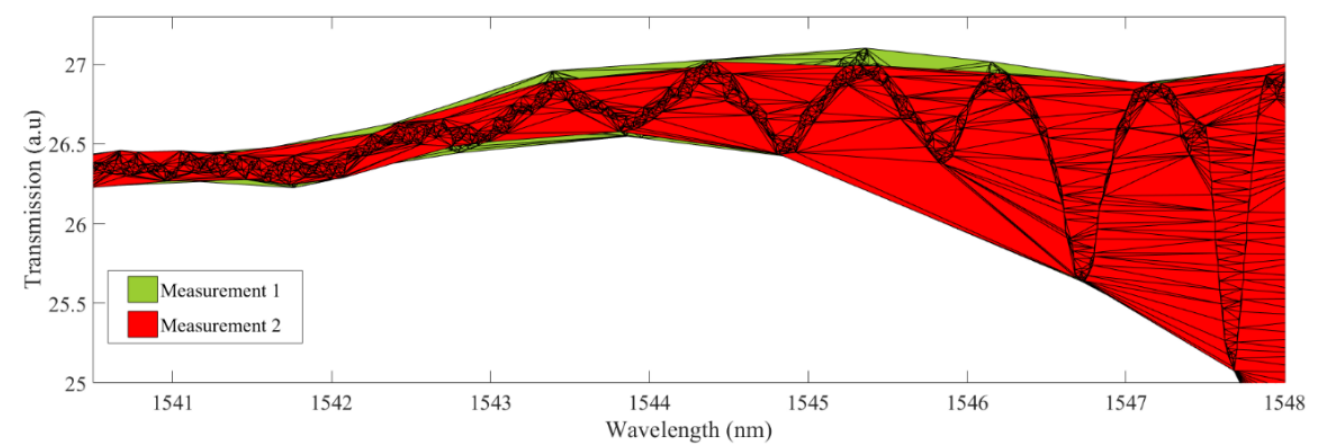

Figure 9. Higher cladding resonance peaks of TFBG transmission spectra after D-triangulation using 1.40 RI liquid.

The difference in shape between the convex hulls is irrelevant for the area calculation as different shapes can produce the same area value. However, here, the dashed rectangles indicate zones in which one of the convex hulls incorporates the second. At still lower wavelengths, the peaks are not detectable anymore and become noise in the spectrum because they are already irradiated out of the OF.

When the TFBG is surrounded by $1.46 \mathrm{RI}$, the higher cladding peaks are not visible; therefore, the curve becomes flatter and the oscillations have smaller amplitudes. In Figure 10, no convex hull incorporates the other one. Hence, this demonstrates that, missing 
the effect of the higher cladding modes, the difference between the areas (and the standard deviation) is smaller with respect to when the TFGB was immersed in 1.40 RI liquid.

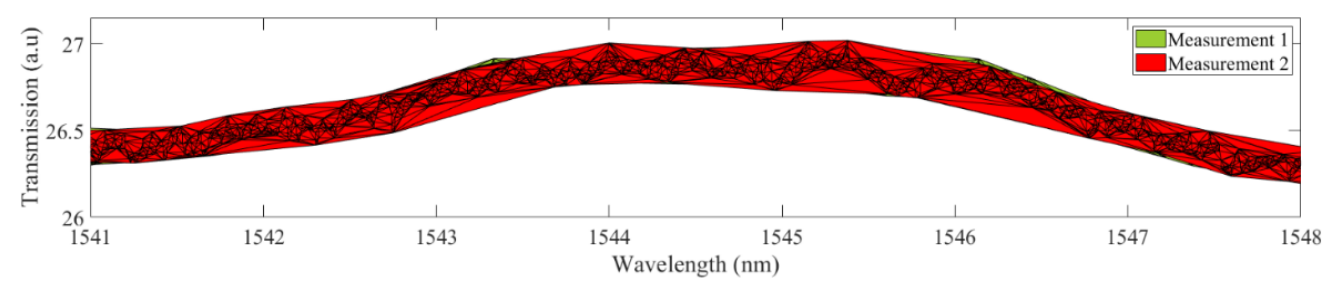

Figure 10. Higher cladding resonance peaks of TFBG transmission spectra after D-triangulation using 1.46 RI liquid.

\section{Influence of the $\alpha$-Shape Parameter on the Stability}

In the previous section, the higher order cladding modes were assumed to create area differences between two TFBG spectra recorded during the immersion in the same RI. However, the accuracy of the interrogation system in scanning the light wavelength transmitted by the Bragg gratings can influence the area measurement. In fact, the wavelength scanning and the power detection accuracy of the interrogator determine the exact positioning of the recorded data points in the spectrum, from which triangulation returns the envelope area.

This means that, when using the same RI at a fixed temperature, two TFBG spectra can have some data points placed in different positions along the wavelength and power transmission range, and, as a consequence, the envelope area could be different, thus, increasing the standard deviation. Since the D-T demodulation technique depends strongly on the $\alpha$-shape parameter, which determines the dimensions of the admissible triangles during the spectrum triangulation, in this section, an analysis was performed of the scope of the $\alpha$ value in reducing the standard deviation (Table 2).

Table 2. Values of standard deviation for the 10 set points at each RI when considering several $\alpha$-parameters.

\begin{tabular}{|c|c|c|c|c|c|c|}
\hline$\alpha$ & 1 & 2 & 3 & 4 & 5 & 6 \\
\hline RI & & & & & & \\
\hline 1.40 & 0.0078 & 0.006 & 0.006 & 0.0072 & 0.0084 & 0.007 \\
\hline 1.41 & 0.005 & 0.0046 & 0.005 & 0.0056 & 0.0047 & 0.0086 \\
\hline 1.42 & 0.0068 & 0.0061 & 0.0057 & 0.0057 & 0.007 & 0.0068 \\
\hline 1.43 & 0.004 & 0.0028 & 0.004 & 0.0033 & 0.0048 & 0.0034 \\
\hline 1.44 & 0.003 & 0.0030 & 0.0032 & 0.0024 & 0.003 & 0.0028 \\
\hline 1.45 & 0.0048 & 0.0043 & 0.0041 & 0.0047 & 0.0089 & 0.0071 \\
\hline 1.46 & 0.0009 & 0.0006 & 0.0006 & 0.0017 & 0.0015 & 0.0012 \\
\hline 1.47 & 0.00076 & 0.0014 & 0.0011 & 0.0006 & 0.00056 & 0.0033 \\
\hline 1.48 & 0.0020 & 0.0024 & 0.0016 & 0.0017 & 0.0023 & 0.0048 \\
\hline 1.49 & 0.0020 & 0.0018 & 0.0019 & 0.0016 & 0.0027 & 0.0025 \\
\hline 1.50 & 0.0016 & 0.0012 & 0.002 & 0.002 & 0.0018 & 0.0013 \\
\hline 1.51 & 0.0013 & 0.0014 & 0.0013 & 0.0012 & 0.0015 & 0.0026 \\
\hline 1.52 & 0.0012 & 0.0016 & 0.0023 & 0.0018 & 0.0018 & 0.0027 \\
\hline 1.53 & 0.00087 & 0.0010 & 0.0008 & 0.0007 & 0.0015 & 0.0035 \\
\hline 1.54 & 0.00062 & 0.0008 & 0.0008 & 0.0007 & 0.001 & 0.0017 \\
\hline 1.55 & 0.0008 & 0.0016 & 0.0013 & 0.0013 & 0.00177 & 0.00085 \\
\hline 1.56 & 0.0013 & 0.0010 & 0.0011 & 0.00082 & 0.00114 & 0.00188 \\
\hline 1.57 & 0.0011 & 0.0007 & 0.0008 & 0.0016 & 0.0015 & 0.00389 \\
\hline 1.58 & 0.0012 & 0.0022 & 0.0010 & 0.0013 & 0.0014 & 0.003 \\
\hline 1.59 & 0.0006 & 0.0006 & 0.0007 & 0.0008 & 0.0009 & 0.0028 \\
\hline 1.60 & 0.0015 & 0.0018 & 0.0014 & 0.0018 & 0.002 & 0.00296 \\
\hline
\end{tabular}

Figures in bold are the smallest standard values in their own row. 
As Table 2 shows, there was no optimum $\alpha$-parameter to minimize the standard deviation for all the RIs. However, it is also observable that higher $\alpha$ values did not have a positive effect on the measurement deviation. In general, small $\alpha$ values decreased $\sigma$, as the triangulation is made with smaller triangles. This reduces the probability to incorporate, in the area calculation, parts of spectrum generated by points that are not accurately detected during the interrogation of the TFBG sensor. Furthermore, though some $\alpha$ values make $\sigma$ smaller than others, the values are closer to make the error negligible when $\alpha$ is between 1 and 4 , thus, obtaining a correlation curve or RI measurement with a good approximation.

\section{Influence of the $\alpha$-Parameter on the Resolution of the D-T Demodulation Technique}

To fully characterize the D-T technique, the last performance parameter to be analysed is the resolution. Substantially, the RI resolution of the TFBG is dependent on the fitting correlation function obtained from the correlation curve after the calibration. In turn, the correlation curve is tied to the $\alpha$ value used to perform the triangulation. For this reason, a specific $\alpha$ can improve the RI resolution of the TFBG in relation to the chosen fitting correlation function. The FBG interrogator can improve or decrease the TFBG resolution based on its scanning wavelength and power resolution. Considering the interrogation system described in Section 3, here, the resolution is analysed by considering several orders of fitting correlation functions obtained for different $\alpha$ values.

The first step is to find the correlation curve for several $\alpha$ from the mean values obtained from the 10 correlation points of each RI. The curves are shown in Figure 11 as the cubic-spline interpolation; it is possible to appreciate that different $\alpha$ parameters generate differences between the curves. However, since, for all the $\alpha$ values, the mean point at 1.46 is the minimum, then the RI range is split into two subintervals 1.40-1.46 and 1.46-1.6 for a better presentation. At this point, the study resolution can be performed; initially the analysis is focused only considering $\alpha=2$ and then also for the other $\alpha$ values.

Hence, first of all, several fitting functions are obtained for the mean correlation points (reported in Figure 12 with $\pm \sigma$ cubic-spline curves). From these functions, the minimum measurable RI step can be obtained by taking into account the minimum step amplitude power detection of the FBG interrogator system. However, the FBG interrogator resolution, given in $\mathrm{dB}$, is not constant along the power amplitude once the spectrum is converted into a linear scale. Therefore, to have the worst measurement condition, the maximum interrogator resolution is taken by considering the entire $\mathrm{dB}$ power amplitude range of the TFBG spectrum. Hence, the minimum step area is then calculated and normalized with respect to the reference area value, and the resolution can be obtained along the fitting functions on both the sub-intervals as shown in Figure 13.

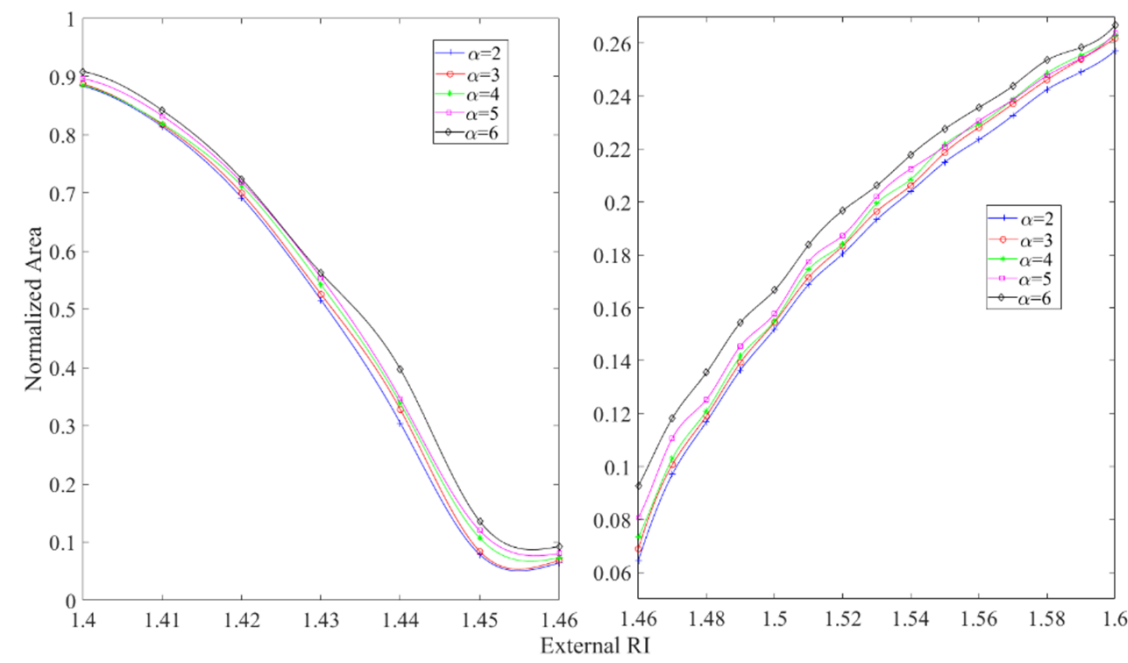

Figure 11. Correlation curves of the mean points for several $\alpha$ values. 


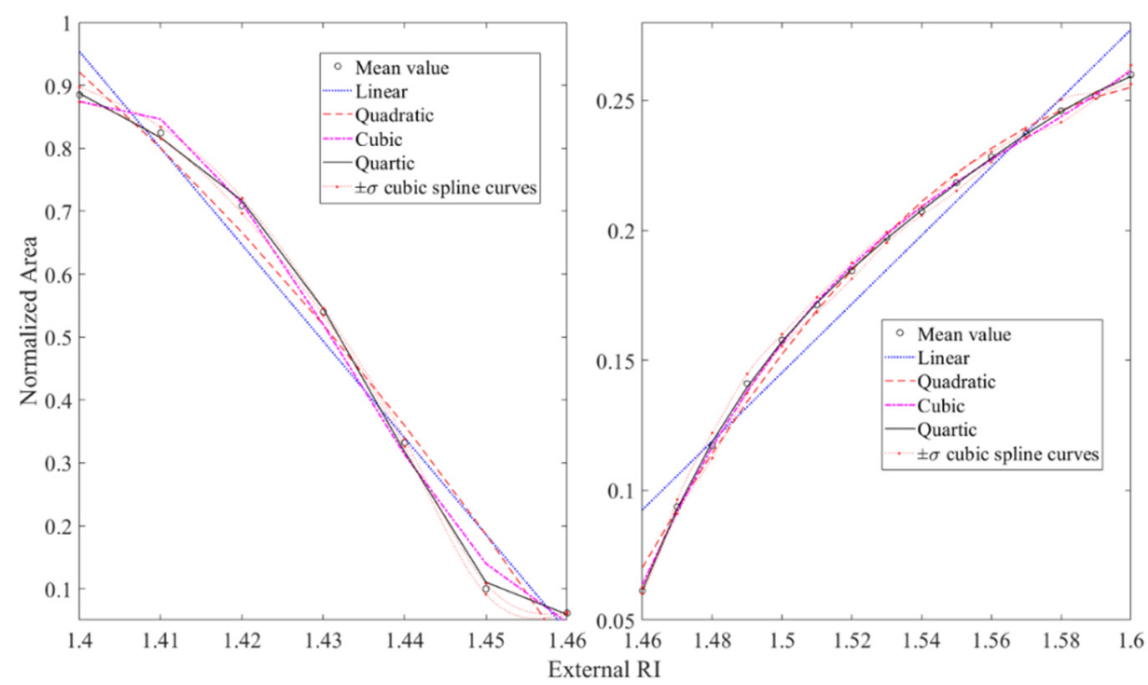

Figure 12. Fitting correlation functions with $\alpha=2$ along the RI range.

In Figure 13, the evolution of the resolution shows that certain degrees of fitting function allow for a finer resolution in some RI ranges. This variation of the resolution is caused by a change in the local slope of the fitting functions, which can vary the minimum RI detectable step correlated with the minimum area variation in the spectrum. Clearly, a linear fitting function would offer the same RI resolution along the entire working range, as its slope is constant. However, while this choice may appear convenient, we also consider that the constant resolution low-degree polynomial functions have a fitting error greater than those at higher order though these can risk being over-fitted. Hence, the accuracy of the measurement would be compromised, though the resolution would be finer and more stable along the RI range.
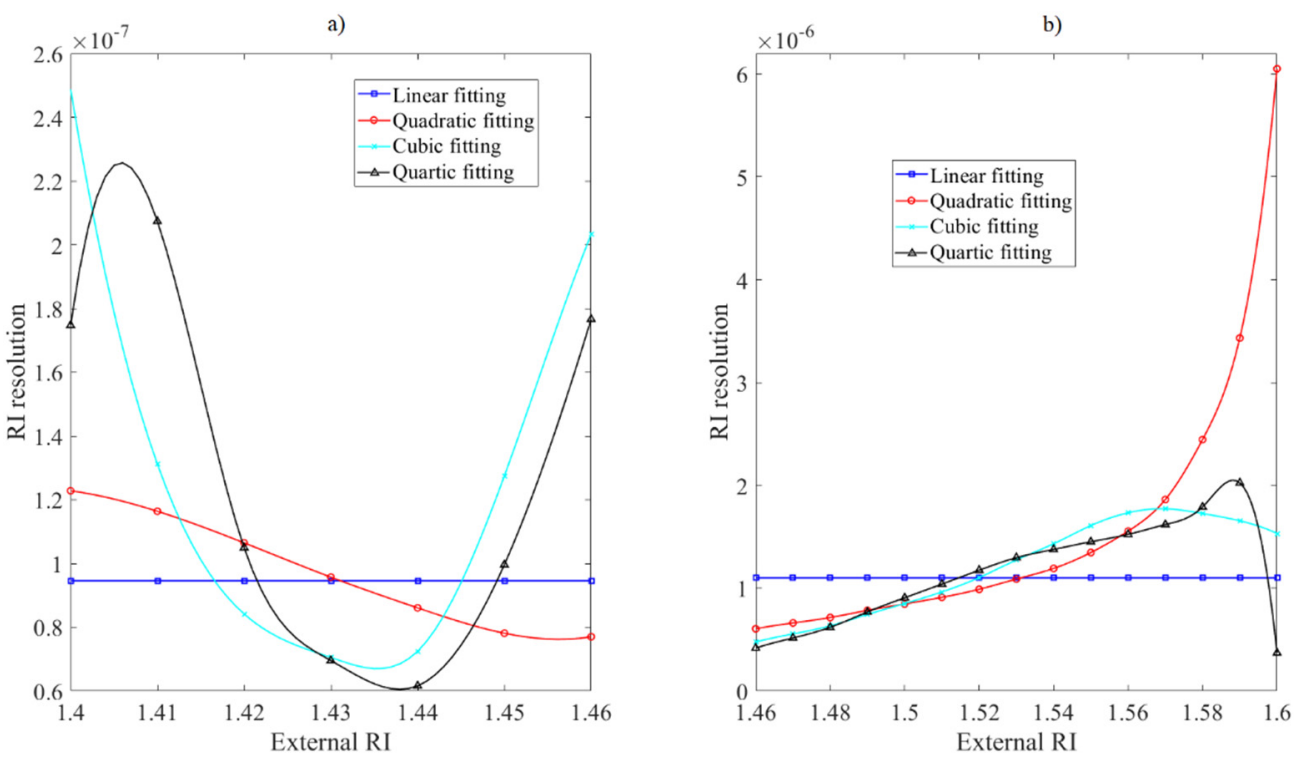

Figure 13. Resolution trends, in RI ranges (a) 1.40-1.46 and (b) 1.46-1.60, for different fitting functions with $\alpha=2$.

The same kind of analysis is made by considering several $\alpha$ values. Therefore, as previously, the fitting functions are calculated and also the associated RI resolution. To have a complete point of view, the goodness of the fitting of each function, associated to the used $\alpha$ value, is reported in Table 3 as the R-square error $\left(R^{2}\right)$.

The resolution trends for the different degrees of fitting functions are reported in Figure 14 for the 1.40-1.46 RI range and Figure 15 from 1.46 to 1.60. An important aspect 
can be observed by comparing the graph of the two RI sub-intervals for the same fitting function. Considering the linear correlation function (Figures 14a and 15a), the same $\alpha$ value does not allow us to obtain the finest possible resolution in both the RI sub-ranges. The same behaviour is observable by comparing the curves of the other fitting functions, where the trends are also different when considering the different monotonous nature of the correlation points from which the fitting functions are generated. However, in general, the resolution variation along the RI ranges is restrained, so that an average value can be used.

Table 3. $\mathrm{R}^{2}$ error of the $\mathrm{i}$-th degree fitting functions in relation to the $\alpha$ parameter used during the D-triangulation.

\begin{tabular}{cccccccc}
\hline & \multicolumn{7}{c}{$\boldsymbol{\alpha}$} \\
\cline { 3 - 8 } & & RI Range & $\mathbf{2}$ & $\mathbf{3}$ & $\mathbf{4}$ & $\mathbf{5}$ & $\mathbf{6}$ \\
\hline \multirow{2}{*}{ Linear } & $1.40-1.46$ & 0.9710 & 0.9675 & 0.9697 & 0.9717 & 0.9731 \\
& $1.46-1.60$ & 0.9520 & 0.9554 & 0.9603 & 0.9605 & 0.9656 \\
\cline { 2 - 8 } Fitting order & \multirow{2}{*}{ Quadratic } & $1.40-1.46$ & 0.9763 & 0.9755 & 0.9796 & 0.9867 & 0.9873 \\
\cline { 2 - 8 } & & $1.46-1.60$ & 0.9953 & 0.9963 & 0.9971 & 0.9975 & 0.9983 \\
\cline { 2 - 8 } & \multirow{2}{*}{ Cubic } & $1.40-1.46$ & 0.9952 & 0.9937 & 0.9951 & 0.9952 & 0.9946 \\
& \multirow{2}{*}{ Qunction } & $1.46-1.60$ & 0.9992 & 0.9993 & 0.9995 & 0.9995 & 0.9995 \\
\cline { 2 - 8 } & \multirow{2}{*}{ Quartic } & $1.40-1.46$ & 0.9993 & 0.9989 & 0.9994 & 0.9983 & 0.9976 \\
& $1.46-1.60$ & 0.9998 & 0.9998 & 0.9997 & 0.9997 & 0.9997 \\
\hline
\end{tabular}

The only exception to this description is represented by the trend reported in Figure 15b in the case of a quadratic fitting. Indeed, here, all the curves have substantially the same amplitude and slope until 1.57, where they take different exponential growth rates. Of all the graphs of Figures 14 and 15, the highest deviation is here (Figure 15b) achieved for the fitting curve obtained with $\alpha=3$ where the resolution at 1.60 is an order of magnitude higher than the same at 1.46. At this point, the TFBG demodulation technique based on the D-T is well characterized for the fundamental measurement performance (stability or repeatability, accuracy and resolution).

Furthermore, the application of the TFBG sensor as a dual- or three-parameter sensor can be performed by knowing a priori the strain cross-sensitivity influence on the RI measurements. From the $\alpha$-parametric analysis of the stability and sensing performance, it is possible to assert that there is no unique choice regarding the best demodulation configuration of the TFBG spectrum for RI measurements. Indeed, the $\alpha$-parameter and the fitting correlation function should be chosen in relation to the resolution, accuracy, goodness/error of the fitting, RI range to measure, computational nimbleness and type of sensor response (linear, quadratic etc.).

To have a practical example, an advanced application for the TFBG sensors regards their embedding in composite materials to monitor the resin degree of cure by measuring its RI. The range of interest for most of the resin is greater than 1.46 (in particular 1.50-1.65) [24]. In this context, since the RI variations can be strong due to the temperature profile used to cure the composite, a fine resolution and stability of the measurements is required to achieve the full RI trend history during the curing step. From Figure 15, the linear, cubic and quartic fitting functions offer the best resolutions.

However for higher degree functions, the resolution trend evolves along the RI range, while the linear fitting correlation guarantees a good and constant resolution (especially for $\alpha=4$ ) along the entire range though its accuracy is lower than the other functions due to the higher $\mathrm{R}^{2}$. In conclusion, taking into account the above considerations, it is possible to assert that the demodulation configuration is a trade-off decision based on the balance between the measurements stability (repeatability), sensing performance (resolution and accuracy) and TFBG application (RI range). 

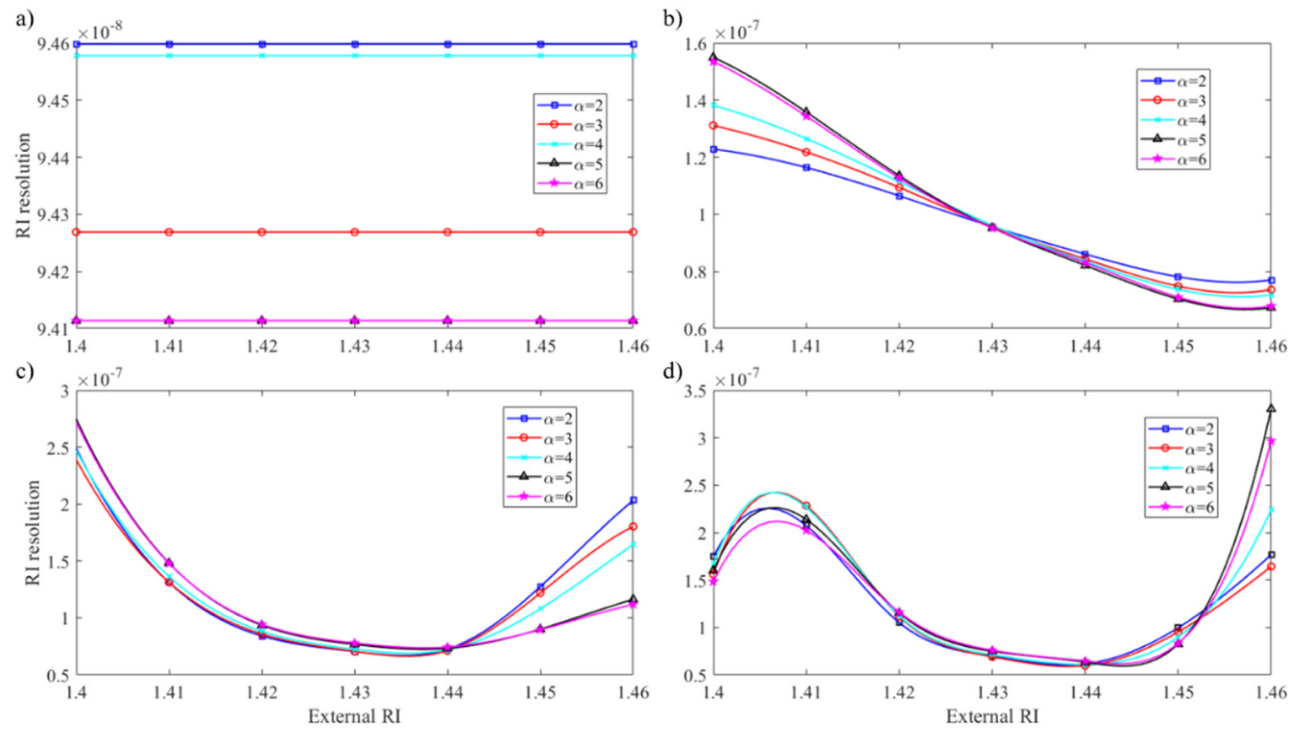

Figure 14. RI resolution for (a) linear, (b) quadratic, (c) cubic and (d) quartic fitting functions with different $\alpha$ along the $1.40-1.46$ RI range.
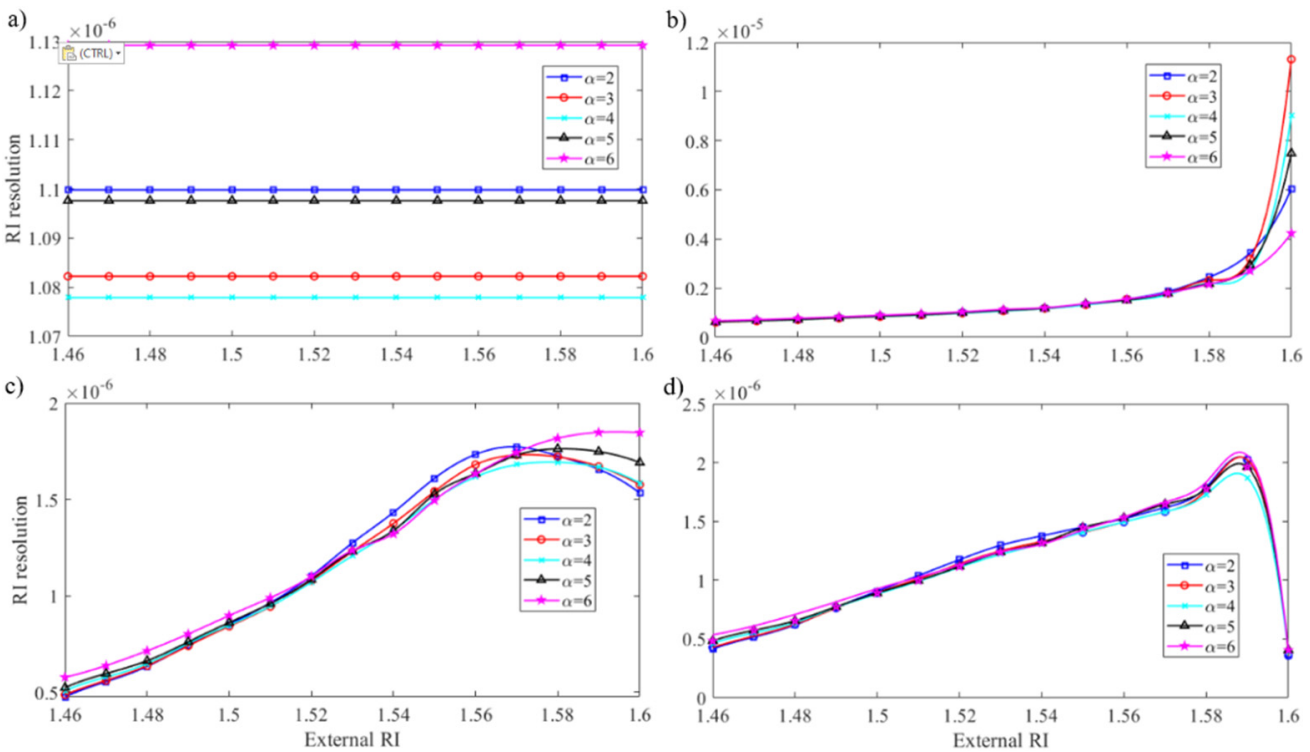

Figure 15. RI resolution for (a) linear, (b) quadratic, (c) cubic and (d) quartic fitting functions with different $\alpha$ along the 1.46-1.60 range.

\section{TFBG Refractometric Properties}

This section is dedicated to the TFBG spectrum behaviour when partially immersed in an RI liquid. As mentioned, the cladding modes are irradiated out from the OF when the external RI matches their effective RI. In particular, the decay of the resonance peaks is much stronger when the surrounding RI is closer to that of the cladding layer. Therefore, total immersion of the sensor in a RI liquid involves a decrease of the total power transmitted from the TFBG and a drastic change in its spectrum, which are exploited to use the TFBG as refractometer. However, to the knowledge of the authors, no study has been reported regarding the evolution of the TFBG transmission spectrum when the Bragg gratings are partially surrounded by a medium with a different RI along its length.

This aspect is relevant when the TFBG is used as refractometer in monitoring applications for the manufacturing and curing of composites materials as the resin flows gradually around the sensor making unknown changes in its spectrum. In Figure 16, a schematic of this case is presented, where the light in the partially submerged TFBG $\left(\right.$ by $\mathrm{RI}_{2}$ ) 
is irradiating out the cladding modes when the effective RI matches (or it is smaller than) the external one. While, in the second part, the cladding modes are internally reflected as the $\mathrm{RI}_{1}$ (of air) is much smaller than the cladding one.

Of course, the mode-coupling system at the interface between two different materials (as cladding-air or cladding-oil) is more complex than the one just described, like the presence of cladding resonance peaks for external RIs higher than the cut-off RI. However, the model here presented is a simple but effective method that is useful to understand the behaviour of the tilted FBG in this unconventional case. Focusing on the transmitted spectrum of the TFBG, the amplitudes of the resonance peaks are obtained for the contribution of each single tilted Bragg grating imposed through the permanent modulation of the core RI profile during sensor manufacturing.

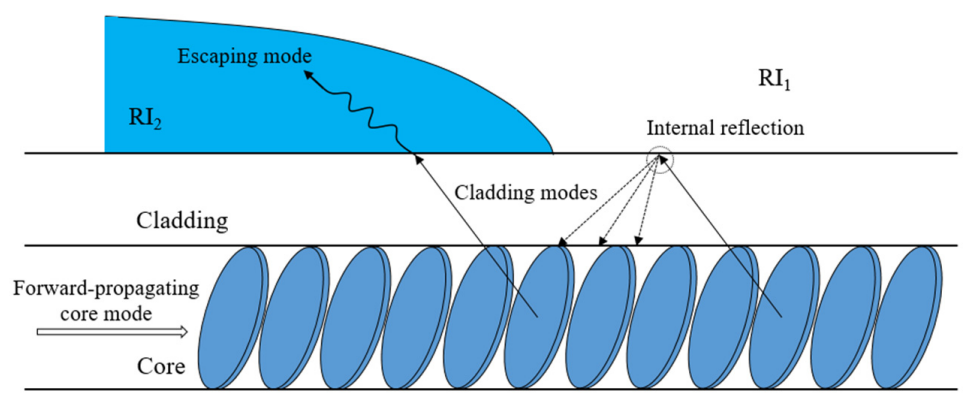

Figure 16. Schematic of a TFBG internal structure surrounded by two different RIs.

This can be demonstrated considering the single Bragg resonance peak of a standard Bragg grating, whose maximum reflectivity $\left(\mathrm{r}_{\max }\right)$ and transmission $\left(\mathrm{t}_{\max }\right)$ is given by [1]:

$$
\begin{aligned}
& r_{\max }=\tanh ^{2}(\kappa \mathrm{L}), \\
& \mathrm{t}_{\max }=\tanh ^{2}(\kappa \mathrm{L}),
\end{aligned}
$$

where $\mathrm{k}$ is the "ac" coupling coefficient and $\mathrm{L}$ is the length of the Bragg grating. Following the equations, the resonance amplitude increases with the FBG length as more Bragg gratings effectively contribute to the coupling of the light. As mathematically treated in the coupled-mode theory [25], the same mechanism is valid also for the generation of the cladding resonance peaks in the spectrum of a TFBG sensor, as each tilted grating gives the same contribution to their generation in the transmitted spectrum, and hence longer TFBGs produce greater resonance amplitudes.

When considering this aspect, when part of the TFBG sensor is surrounded by a medium with a different RI, the mode-coupling locally changes and some cladding modes are irradiated out from the $\mathrm{OF}$, influencing the amplitude and the wavelength of the cladding modes resonances and, hence, the spectrum shape and the transmitted TFBG power. Therefore, to investigate the effects on the transmitted signal of a possible partial immersion along the TFBG length, the situation shown in Figure 16 was replicated using the setup of Figure 3 and a camera with a high frame rate (up to $55 \mathrm{fps}$ ) was positioned to record the different degrees of longitudinal immersion.

The immersion degree was quantified in the TFBG length submerged in the liquid by measuring it digitally from the pictures acquired with the camera. The dipping rate along the axis of the OF sensor was controlled using the vertical movement of the translation stage on which the polycarbonate block was placed. The surface tension in combination with the distance of the RI liquid (1.46) from the OF was used to control the flow along the length of the OF sensor. The TFBG spectra were recorded every $3 \mathrm{~s}$ of the experiment where gradually the liquid enveloped the TFBG, and simultaneously the pictures were taken for $1 \mathrm{~s}$ across each spectrum acquisition. In Figure 17, seven images show the increasing immersion degrees in $1.46 \mathrm{RI}$ oil of the OF during the experiment where the input light inside the OF comes from the left side; hence, the light transmitted from the TFBG flows on the right side. 
The first image is the starting condition when the RI liquid is in contact with the OF but not yet with the section where the TFBG is written; here, the transmitted spectrum is the same as that obtained when the sensor is surrounded by air. Therefore, from this point, the liquid is forced to flow along the TFBG length by exploiting the oil surface tension and decreasing the distance between the $\mathrm{OF}$ and the polycarbonate block through the vertical translation stage. In this way, increasing immersion degrees are obtained, until Figure $17 \mathrm{~g}$ where the TFBG is fully wetted by the RI liquid.

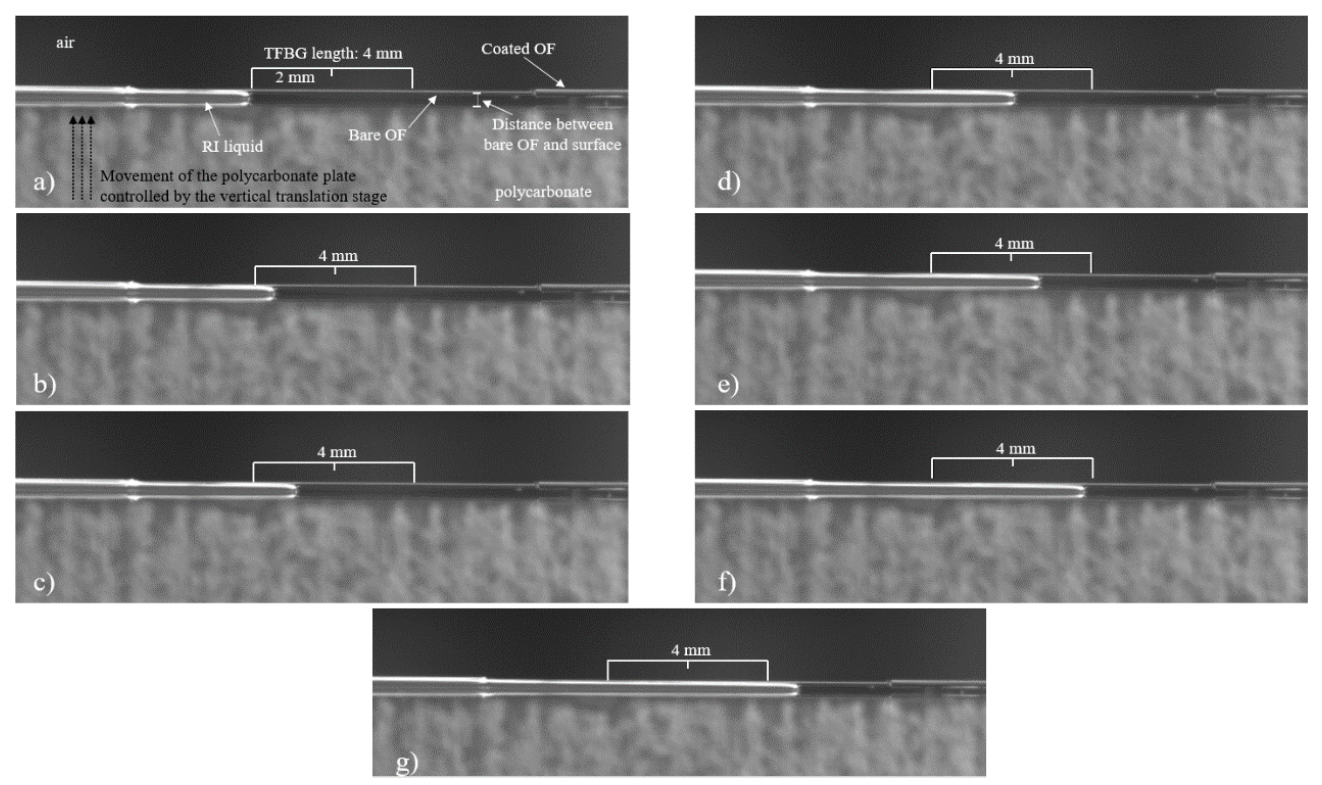

Figure 17. Frames sequence (a-g) during the gradual immersion of the TFBG sensor in a 1.46 RI oil.

The spectra recorded at each picture of Figure 17, were used to analyse the trend of the cladding resonance envelope area with the propagation of the RI liquid along the sensor axis. The calculation of the envelope area was performed using the D-T demodulation technique introduced in the previous sections. Once the value of the envelope area was obtained, it was normalized with respect to the spectrum of the unchanged TFBG. Figure 18 shows the percentages of the envelope area calculated from the TFBG spectra obtained experimentally versus with the submerged TFBG length. From Figure 18, the envelope area of the cladding modes resonance was observed to decrease with the forward propagation of the RI liquid.

The blue dots are the normalized areas obtained from the TFBG spectra recorded during the experiment, and the letters refer the points to the images of Figure 17 (where Figure $17 \mathrm{a}$ is the reference at $100 \%$ ). With the progress of the RI liquid flow front along the TFBG axis, a higher RI surrounds more Bragg grating lengths. Therefore, the envelope area decreases because the cladding modes coupled in those gratings are irradiated out of the $\mathrm{OF}$, not giving any contribution to the transmitted spectrum. The blue dot point $g$ in Figure 18 refers to the TFBG fully incorporated in the liquid (Figure 17g); here, the area is considered to be at $0 \%$, as all the cladding modes reflections contributing to the spectrum are suppressed.

A linear correlation between the envelope area trend and submersion length was expected by considering the proportional relation between the Bragg gratings and the resonances peaks in the TFBG spectrum. However, the linear fitting was obtained with an unexpected R-squared error of 0.9802. This indicates that, although the TFBG submersed length could be determined digitally measuring the flow front propagation of the oil, it did not correspond with the real immersion length. Hence, each estimated immersion length contains an error due to the surface tension and capillarity forces between the RI liquid and $\mathrm{OF}$. 


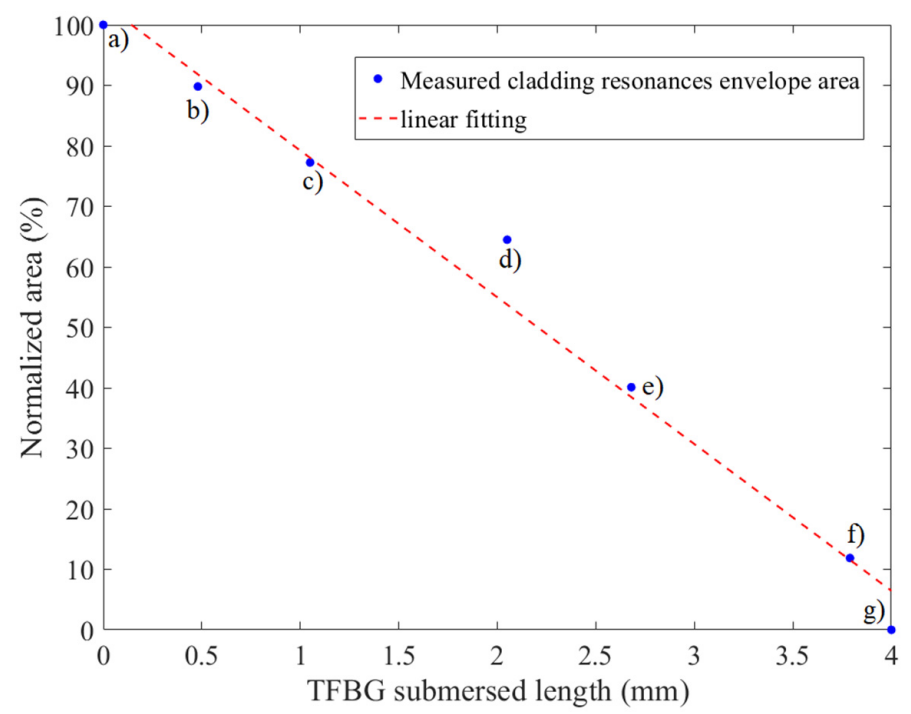

Figure 18. Trend of normalized cladding modes area versus the TFBG submerged length.

An accurate estimation of the immersion length can be performed using the envelope area obtained from the TFBG spectrum. Indeed, as described previously, the contribution to the resonance peaks amplitudes depends proportionally on the TFBG length, independently from the resulting coupling-mode. This means that the longer the immersion length is, the greater the decreasing of the envelope area is. Especially, as each Bragg grating of the TFBG gives the same contribution to the spectrum, this decrease is linear with the immersion length.

Therefore, considering the two extreme dipping points and the TFBG length, a linear trend relation between the normalized area and immersion length can be obtained. By solving the linear function with the normalized area values, the immersion lengths are calculated. In Figure 19, the digitally measured immersion lengths are reported together with those calculated using the envelope area of the TFBG and its linear relation with the immersion length. For a clearer comparison, the immersion length values obtained with both the techniques are listed in Table 4 in relation to the images of Figure 17.

Table 4. The TFBG immersion length in the RI liquid estimated from the images of Figure 17 and through the envelope areas.

\begin{tabular}{ccccccccc}
\hline \multicolumn{2}{c}{ Images of Figure 17} & $\mathbf{a}$ & $\mathbf{b}$ & $\mathbf{c}$ & $\mathbf{d}$ & $\mathbf{e}$ & $\mathbf{f}$ & $\mathbf{g}$ \\
\hline \multirow{2}{*}{$\begin{array}{c}\text { TFBG immersion } \\
\text { length }(\mathrm{mm}) \text { estimated }\end{array}$} & Digitally & 0 & 0.48 & 1.05 & 2.05 & 2.61 & 3.73 & $\geq 4$ \\
\cline { 2 - 9 } & Envelope area & 0 & 0.408 & 0.910 & 1.421 & 2.396 & 3.525 & $\geq 4$ \\
\hline
\end{tabular}

Table 4 shows that the immersion values estimated digitally are greater than those calculated using the envelope area provided by the TFBG spectrum. The reason is because the digital measurement is performed considering the flow front of the liquid. However, the flow front does not correspond with the real immersion length as the tension surface forces between the immersed OF and liquid compress the liquid front, making this lower than the OF.

To conclude the investigation of the experimental data obtained from the progressive immersion of the TFBG sensor, the recorded spectra at each submersion length are shown in Figure 20. The first observable fact is the change of the upper and lower resonance peak amplitudes along the working wavelength with the increase of the immersed TFBG length. As expected, the Bragg and Ghost peaks do not undergo any changes in amplitude and in wavelength. As a consequence, these results further demonstrate that the coupling-mode in the inner OF layer is not influenced by a variation of the external RI around the partial or total length of the TFBG sensor. 


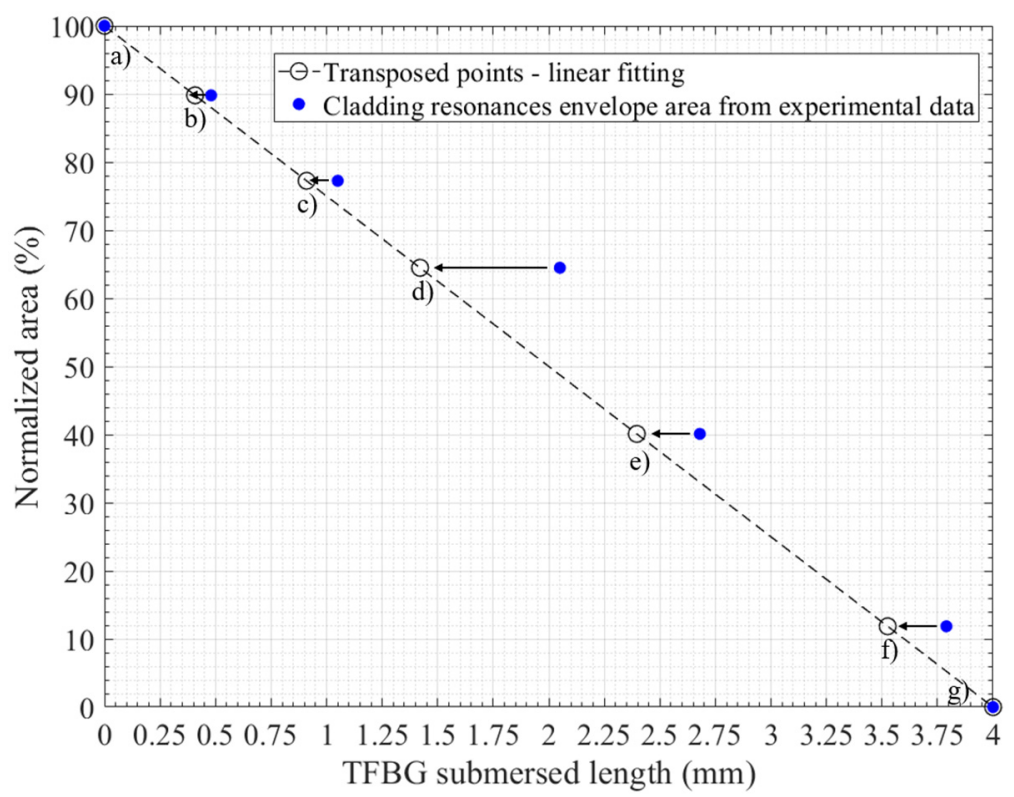

Figure 19. Correction through the TFBG (black circles) of the immersion lengths measured digitally from Figure 18 (blue dots).

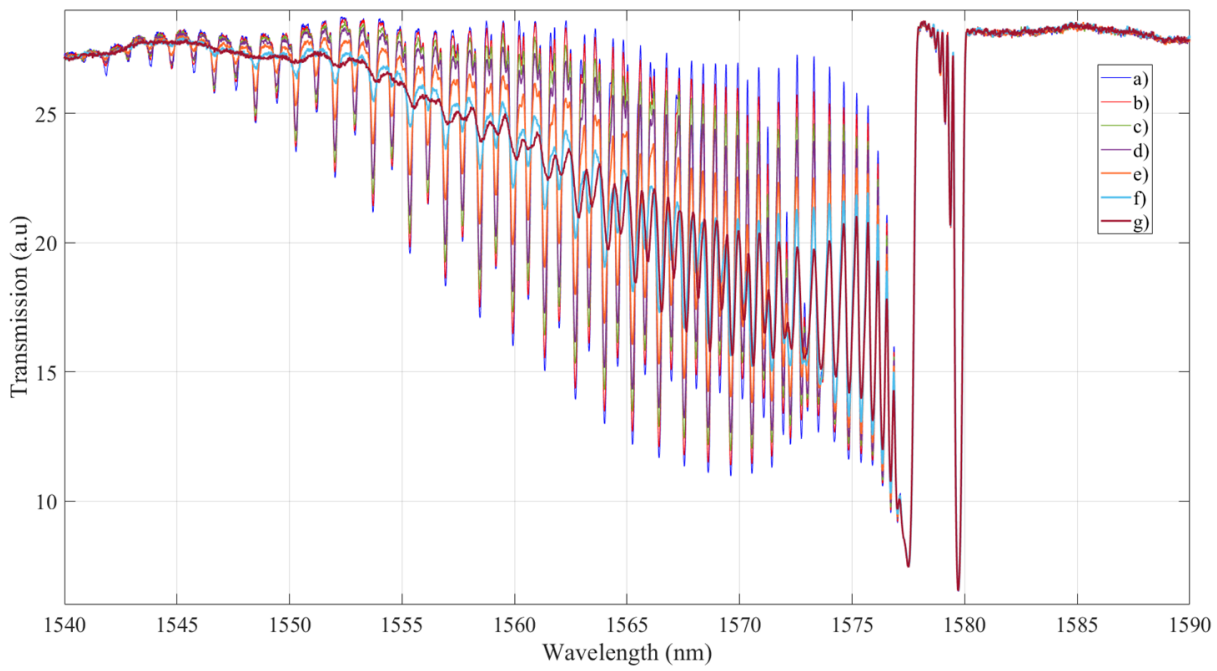

Figure 20. TFBG spectra acquired during the experiment and related to the snapshots of Figure 17.

By focusing on the cladding resonance peak wavelengths, in addition to the amplitude variation, it is also possible to note a small red shift of these peaks once the TFBG is fully wet by the oil. This behaviour is well known and has already been exploited to calculate the RI [13]. Zooming in to Figure 20 to certain upper and lower cladding peaks, the dynamic of this wavelength variation can be analysed. Figure 21 shows the decay of the transmitted cladding peak that occurs as the liquid flows along the sensor. It is interesting to note that, the peak remains substantially at its nominal wavelength $(1568.6 \mathrm{~nm})$, but it becomes broader, especially on the side of higher wavelengths.

When the sensor is fully surrounded by the RI liquid, the peak appears completely shifted. This effect is due to the dependency of the modes to their effective RI during the coupling as Equation (2) reports. When the liquid covers part of the TFBG, the remaining cladding modes propagate with a different effective RI due to the variation of the external one. Hence, as Equation (2) shows, their resonance wavelength changes and that means their contribution to the amplitude is more broadly distributed with respect to when the TFBG is uniformly surrounded. 


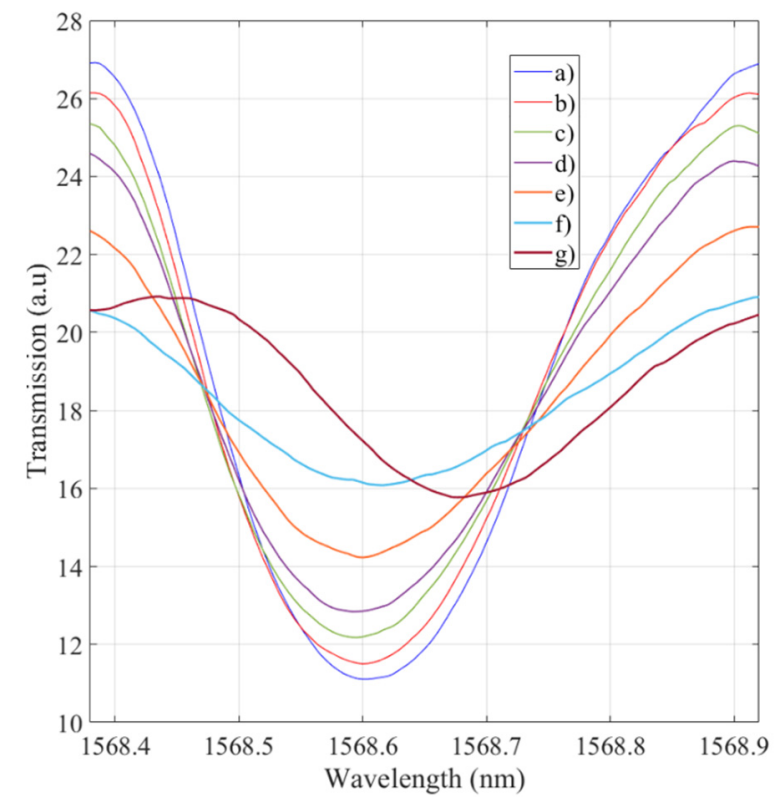

Figure 21. Zooming of the TFBG spectra on certain cladding resonance upper and lower peaks.

A proof of this physical explanation can be found by comparing the peak of Figure 21 in the immersion conditions $\mathrm{f}$ and $\mathrm{g}$ (Figure 17). In particular, the peak in condition $g$ (TFBG fully immersed), is visibly more defined and has a smaller bandwidth with respect to that obtained at immersion condition $\mathrm{f}$. The complete shifting of the cladding resonance peaks can be used to identify a fully or partial immersion condition of the TFBG sensor.

Using the TFBG spectra of Figure 20, once the Delaunay triangulation was performed, the envelope areas of the upper and lower cladding resonance peaks were obtained, and they are shown overlapped in Figure 22. Since the spectrum associated to the first snapshot has the greater envelope area, the union of all the underlying areas represents it until the blue. This indicates that the envelope area number two is the overlap of all the areas until the red (removing the blue regions), and the same is valid for each successive area. As expected from the previous description, the reduction of the envelope areas is observable with the immersion of the TFBG, where each subsequent area is gradually contained in the previous ones, thus, creating a sort of 'funnel' effect between them.

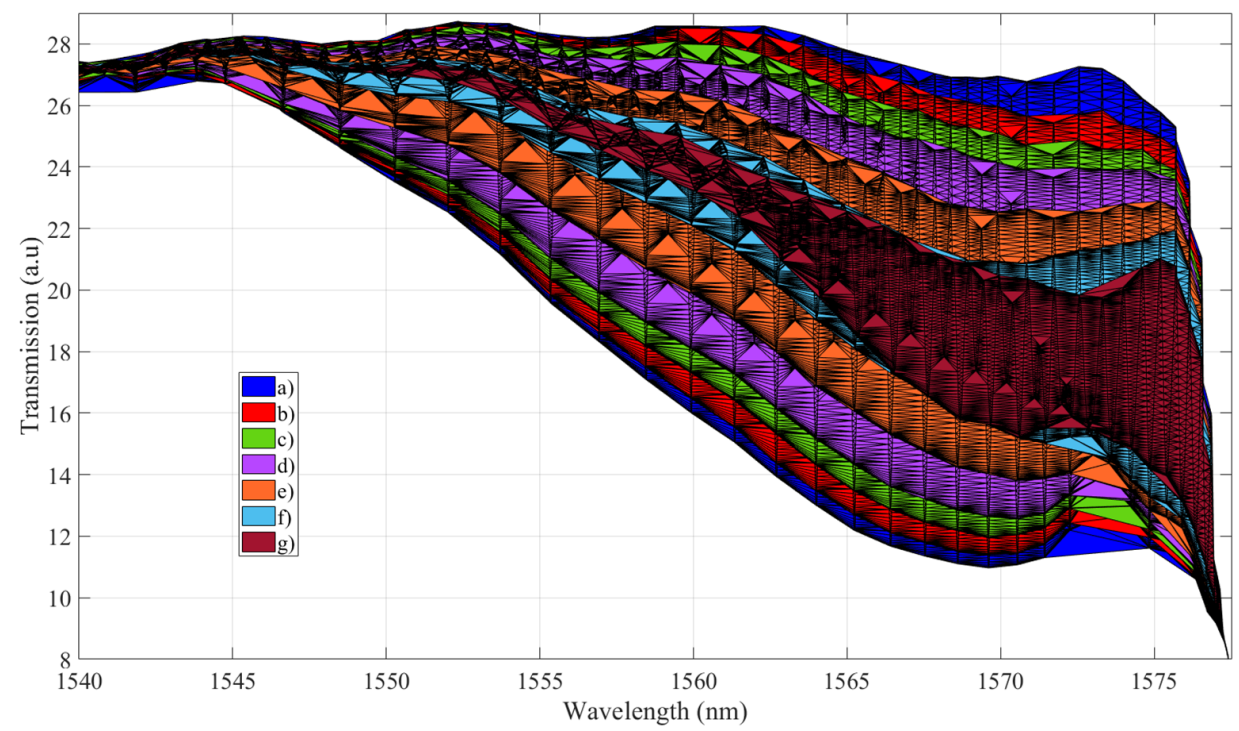

Figure 22. Envelope areas of the cladding modes resonance peaks of the spectra shown in Figure 20 using the D-T demodulation technique. 


\section{Conclusions}

A complete characterization of the measurement performance of the TFBG sensor demodulated with the Delaunay triangulation technique as a refractometer was achieved in this work. The stability of the measurements and the resolution were widely analysed for several $\alpha$-values, obtaining, in certain RI ranges, a resolution less than $10^{-7}$ independently of the parameter used to perform the triangulation and from the fitting correlation function. The stability was analysed not only numerically but also by focusing on the high order cladding resonance peaks, and the reasons for the RI measurement fluctuations were investigated and described.

The alteration of the measurement performance was investigated in the case of dualparameter TFBG sensors for simultaneous strain and RI detection. A change in the envelope area of the cladding resonance peaks was present due to the applied strain on the TFBG. However, the change of the envelope area was not proportional with the deformation and reached a maximum for the lower RI and particular value of strain due to a particular configuration of the peaks in the spectrum. The RI deviation from the unperturbed condition was calculated and corresponded to $0.77 \%$ of the RI reference value. However, as previously described, this error includes the strain influence, temperature fluctuation, fitting error and precision of the FBG interrogator device and was calculated in the worst sensitivity range of the TFBG, which represents the worst measurement conditions.

The last part of the work was dedicated to the investigation of the refractometric properties of the TFBG, where the sensor was gradually immersed in the RI oil. Therefore, the evolution of the envelope area was observed through correlating the variations with the snapshots of the immersion taken during the experiment. From the area evolution, the real TFBG length wetted by the RI oil was calculated and related to the frames sequence. The last step concerned the changing dynamic of the spectral signal during the immersion. In particular, the sequence of the entire spectrum and a zoomed in cladding resonance peak were visualized with the gradual immersion in the oils, showing the dynamics of the shape and wavelength variation. In particular, based on the wavelength shifting of the cladding peaks it was possible to determine independently if the TFBG was fully surrounded by another RI.

This result is of considerable importance if the TFBG is applied to monitor the manufacturing and the curing of composite materials during resin transfer moulding manufacturing processes. Indeed, in exploiting the TFBG refractometric properties as described here, it could be possible to measure important parameters, such as the interlaminate speed of the resin during the infusion, the permeability of the reinforcement fibre layers and the detection of eventual voids as well as to evaluate the infusion state of composites.

To conclude, this work provides a deep and necessary understanding of the TFBG used as a refractometer sensor and demodulated through the D-T demodulation technique, which improved the RI measurement performance of the entire TFBG sensing system.

Author Contributions: Conceptualization, L.F., R.M.G.; methodology, L.F.; software, L.F.; validation, L.F., R.M.G.; formal analysis, L.F.; investigation, L.F.; resources, R.M.G.; data curation, L.F.; writingoriginal draft preparation, L.F.; writing —review and editing, L.F., R.M.G.; visualization, L.F., R.M.G.; supervision, R.M.G.; project administration, R.M.G.; funding, R.M.G. All authors have read and agreed to the published version of the manuscript.

Funding: This research received no external funding.

Institutional Review Board Statement: Not applicable.

Informed Consent Statement: Not applicable.

Data Availability Statement: All the information are reported in the article. No data are available online.

Acknowledgments: We would like to thank our colleague Andrei Anisimov for his contribution in choosing the best TFBG sensor customization and his comments and suggestions during the preparation of this paper. This research was supported by the Operationeel Programma Zuid- 
Nederland (Op-Zuid) Project as part of the Dutch Composite Maintenance Centre (DCMC), supported by the Europees Fonds voor Regionale Ontwikkeling (EFRO) and the North Brabant province of the Netherlands.

Conflicts of Interest: The authors declare no conflict of interest.

\section{References}

1. Erdogan, T. Fiber Grating Spectra. J. Lightwave Technol. 1997, 15, 1277-1294. [CrossRef]

2. Dong, X.; Zhang, H.; Liu, B.; Miao, Y. Tilted Fiber Bragg Gratings: Principle and Sensing Applications. Photonic Sens. 2011, 1, 6-30. [CrossRef]

3. Laffont, G.; Ferdinand, P. Tilted short-period fibre-Bragg-grating- induced coupling to cladding modes for accurate refractometry. Meas. Sci. Technol. 2011, 12, 765-770. [CrossRef]

4. Rajan, G.; Prusty, B.G. Structural Health Monitoring of Composite Structures Using Fiber Optic Methods; CRC Press: Boca Raton, FL, USA, 2003; pp. 238-243.

5. Buggy, S.J.; Chehura, E.; James, S.W.; Tatam, R.P. Optical fibre grating refractometers for resin cure monitoring. J. Opt. A Pure Appl. Opt. 2007, 9, S60-S65. [CrossRef]

6. Shen, C.; Liu, D.; Lian, X.; Lang, T.; Zhao, C.; Semenova, Y.; Albert, J. Microfluidic flow direction and rate vector sensor based on a partially gold-coated TFBG. Opt. Lett. 2020, 45, 2776-2779. [CrossRef] [PubMed]

7. Baldini, F.; Brenci, M.; Chiavaioli, F.; Giannetti, A.; Trono, C. Optical fibre gratings as tools for chemical and biochemical sensing. Anal. Bioanal. Chem. 2012, 402, 109-116. [CrossRef]

8. Guo, T.; Liu, F.; Guan, B.O.; Albert, J. Tilted fiber grating mechanical and biochemical sensor. Opt. Laser Technol. 2016, 78, 19-33. [CrossRef]

9. Melo, L.B.; Rodrigues, J.M.; Farinha, A.S.; Marques, C.A.; Bilro, L.; Alberto, N.; Tomé, J.P.; Nogueira, R.N. Concentration sensor based on a tilted fiber Bragg grating for anions monitoring. Opt. Fiber Technol. 2014, 20, 422-427. [CrossRef]

10. Wang, Q.; Jing, J.; Wang, B. Highly sensitive SPR biosensor based on graphene oxide and staphylococcal protein A co-modified TFBG for human IgG detection. IEEE Trans. Instrum. Meas. 2020, 68, 3350-3357. [CrossRef]

11. Fazzi, L.; Groves, R.M. Demodulation of a tilted fibre Bragg grating transmission signal using $\alpha$-shape modified Delaunay triangulation. Measurement 2020, 166, 108197. [CrossRef]

12. Alberto, N.J.; Marques, C.A.; Pinto, J.L.; Nogueira, R.N. Three-parameter optical fiber sensor based on a tilted fiber Bragg gratin. Appl. Opt. 2010, 49, 6085-6091. [CrossRef]

13. Erdogan, T.; Sipe, J.E. Tilted fiber phase gratings. J. Opt. Soc. Am. 1996, 13, 296-313. [CrossRef]

14. Chan, C.; Chen, C.; Jafari, A.; Laronche, A.; Thomson, D.J.; Albert, J. Optical fiber refractometer using narrowband cladding-mode resonance shifts. Appl. Opt. 2007, 46, 1142-1149. [CrossRef]

15. Fazzi, L.; Valvano, S.; Alaimo, A.; Groves, R.M. A simultaneous dual-parameter optical fibre single sensor embedded in a glass fibre/epoxy composite. Compos. Struct. 2021, 270, 114087. [CrossRef]

16. Cargille Lab. Available online: https://www.cargille.com/refractive-index-liquids/ (accessed on 5 January 2021).

17. Zaber. Available online: https://www.zaber.com/products/linearactuators/NA/noAD/details/NA23C60-T4 (accessed on 5 January 2021).

18. Baker, S.R.; Rourke, H.N.; Baker, V.; Goodchil, D. Thermal Decay of Fiber Bragg Gratings Written in Boron and Germanium Codoped Silica Fiber. J. Lightwave Technol. 1997, 15, 1470-1477. [CrossRef]

19. Kumar, J.; Singh, G.; Saxena, M.K.; Prakash, O.; Dixit, S.K.; Nakhe, S.V. Development and Studies on FBG Temperature Sensor for Applications in Nuclear Fuel Cycle Facilities. IEEE Sens. J. 2020, 21. [CrossRef]

20. Caucheteur, C.; Paladino, D.; Pilla, P.; Cutolo, A.; Campopiano, S.; Giordano, M.; Cusano, A.; Mégret, P. External refractive index sensitivity of weakly tilted fiber Bragg gratings with different coating thicknesses. IEEE Sens. J. 2008, 8, 1330-1336. [CrossRef]

21. Cieszczyk, S.; Harasim, D.; Kisala, P. A novel simple TFBG spectrum demodulation method for RI quantification. IEEE Photonics Technol. Lett. 2017, 29, 2264-2267. [CrossRef]

22. Gang, T.; Liu, F.; Hu, M.; Albert, J. Integrated differential area method for variable sensitivity interrogation of tited fiber bragg grating sensors. J. Lightwave Technol. 2019, 37, 4531-4536. [CrossRef]

23. Chen, C.; Albert, J. Strain-optic coefficients of individual cladding modes of single mode fibre: Theory and experiment. Electron. Lett. 2006, 42, 1027-1028. [CrossRef]

24. Sua, W.F.; Fu, Y.C.; Pan, W.P. Thermal properties of high refractive index epoxy resin system. Thermochim. Acta 2002, 392, 385-389. [CrossRef]

25. Erdogan, T. Cladding-mode resonances in short- and long- period fiber grating filter. J. Opt. Soc. Am. 1997, 14, 1760-1773. [CrossRef] 